\title{
Positive metric currents and holomorphic chains in Hilbert spaces
}

\author{
Samuele Mongodi
}

\begin{abstract}
We present some results concerning currents of integration on finite-dimensional analytic spaces in Hilbert spaces, using the setting of metric currents. In particular, we obtain the characterization of such currents as positive closed $(k, k)$-integer rectifiable currents and solve the boundary problem for holomorphic chains.
\end{abstract}

\section{Introduction}

The theory of currents found deep and important applications in complex analysis and geometry; just to mention a few of them, we recall the characterization of holomorphic chains by King in [9] and by Harvey and Shiffman in [8], the removal of singularities for analytic functions and sets by Shiffman in [15] and the boundary problem for holomorphic chains by Harvey and Lawson in [6], [7].

Such a powerful tool was extended to general metric spaces in [1] and we described some possible applications to complex analysis in [10], specializing the theory in the case of singular complex spaces and complex Banach spaces. In order to gain a wider understanding of the latter, here we turn our attention to holomorphic metric chains in Hilbert spaces, tackling two specific problems: firstly, we consider positive $(k, k)$-integer rectifiable closed metric currents and investigate their link with complex analytic sets; then, we proceed to study the boundary problem for holomorphic (metric) chains and give a positive answer under suitable hypotheses of regularity and of general position.

We organized the material as follows.

After recalling the basic definitions and properties of metric currents and rectifiable sets in metric spaces (from [1], [2]), we summarize the results presented in [10] on the extension of metric currents to complex Banach spaces. The extension of the theory of metric currents to the complex setting forces us to a change of notation: in geometric measure theory it is common to denote the boundary

Mathematics Subject Classification (2010): 32C30, 32V25, $46 \mathrm{G} 20$.

Keywords: Positive currents, holomorphic chains, metric currents, complex Plateau problem. 
of a current $T$ with the symbol $\partial T$, but, as in complex analysis the operators $\partial$ and $\bar{\partial}$ already exist and are widely employed, we preferred to write $d T$ for the boundary of a current, following a convention already established by other papers which employ currents in complex analysis and geometry.

In Section 4, the properties of analytic sets and of the currents of integration associated to them are investigated; in particular, we introduce the concept of positive metric currents, presenting some simple properties, and we obtain a weak analogue of Wirtinger's formula.

The main result of this section is the extension of King's characterization theorem (see [9]) for holomorphic chains to Hilbert spaces.

Theorem 4.12. Let $\Omega \subset H$ be a ball, $S$ be an integer rectifiable current in $\Omega$. Suppose that

1) $\operatorname{supp} d S \cap \Omega=\emptyset$;

2) $S$ is a $(k, k)$ positive current.

Then $S$ can be represented as a sum with integer coefficients of integrations on the regular parts of analytic sets.

In the last section, we turn our attention to maximally complex metric currents and CR-manifolds. We recover a finite-dimensional embedding result and a characterization for maximally complex integration currents.

Finally, we tackle the boundary problem for holomorphic chains in a Hilbert space: we solve the problem with a technical hypothesis on $M$, specifically, the existence of a finite rank projection with transverse self-intersections; we refer to Section 5 for the definition and the discussion of the concept of maximally complex cycle, here we only stress that it is a necessary hypothesis for such a result.

Theorem 5.10. Let $M$ be a compact, oriented $(2 p-1)$-manifold (without boundary) of class $\mathcal{C}^{2}$ embedded in $H$, and suppose that there exists an orthogonal decomposition $H=\mathbb{C}^{p} \oplus H^{\prime}$ such that the projection $\pi: H \rightarrow \mathbb{C}^{p}$, when restricted to $M$, is an immersion with transverse self-intersections. Then, if $M$ is maximally complex (i.e., $[M]$ is an MC-cycle), there exists a unique holomorphic p-chain $T$ in $H \backslash M$ with $\operatorname{supp} T \Subset H$ and finite mass, such that $d T=[M]$ in $H$.

It is worth noting that the Hilbert space hypothesis is not clearly required in these results, but our methods rely heavily on the possibility of estimating norms in terms of coordinates, which is a feature essentially related to the existence of an orthonormal basis.

Some of these results, concerning the boundary problem, have been extended to the non compact case by A. Saracco and the author in [11].

Acknowledgements. I would like to thank prof. Luigi Ambrosio for the fruitful discussions about the geometric measure theory tools employed in the proof of King's result. I would also like to thank the referee for his/her helpful suggestions concerning the presentation of this paper. 


\section{Metric currents}

Let $X$ be a metric space. Let us denote by $\operatorname{Lip}(X)$ the space of complex-valued Lipschitz functions on $X$ and by $\operatorname{Lip}_{b}(X)$ the algebra of bounded complex-valued Lipschitz functions. Following [10], we introduce the spaces

$$
\begin{aligned}
& \mathcal{E}^{k}(X)=\left\{\left(f, \pi_{1}, \ldots, \pi_{k}\right) \mid f \in \operatorname{Lip}_{b}(X), \pi_{j} \in \operatorname{Lip}(X), j=1, \ldots, k\right\}, \\
& \mathcal{E}^{0}(X)=\operatorname{Lip}_{b}(X) .
\end{aligned}
$$

The elements of these spaces are called metric forms.

Definition 2.1 (see Definition 3.1 in [1]). A $k$-dimensional metric current is a functional $T: \mathcal{E}^{k}(X) \rightarrow \mathbb{C}$ satisfying the following:

1) $T$ is multilinear;

2) whenever $\left(f, \pi^{i}\right) \rightarrow(f, \pi)$ pointwise, with uniformly bounded Lipschitz constants, then $T\left(f^{i}, \pi^{i}\right) \rightarrow T(f, \pi)$

3) $T(f, \pi)=0$, whenever there is an index $j$ for which $\pi_{j}$ is constant on a neighborhood of supp $f$;

4) $T$ has finite mass, i.e., there exists a finite Radon measure $\mu$ on $X$ such that

$$
|T(f, \pi)| \leq \prod_{j=1}^{k} \operatorname{Lip}\left(\pi_{j}\right) \int_{X}|f| d \mu \quad \forall(f, \pi) \in \mathcal{E}^{k}(X) ;
$$

the infimum of such measures $\mu$ is called the mass (measure) of $T$.

We will denote by $M_{k}(X)$ the space of $k$-dimensional metric currents on $X$; endowing $M_{k}(X)$ with the mass norm $\|T\|=\mu(X)$, where $\mu$ is the mass measure of $T$, we turn it into a Banach space. We will sometimes write $T(f d \pi)$ for $T\left(f, \pi_{1}, \ldots, \pi_{k}\right)$.

We refer to [1] for a detailed discussion of the general properties of metric currents; here we recall only the main definitions and facts.

Definition 2.2 (see Definition 3.6 in [1]). We say that $T_{h} \in M_{k}(X)$ converge weakly to $T \in M_{k}(X)$ if

$$
T_{h}(f d \pi) \rightarrow T(f d \pi) \quad \forall f d \pi \in \mathcal{E}^{k}(X) .
$$

Definition 2.3 (see Definition 2.3 in [1]). Given $T \in M_{k}(X), k \geq 1$, we define the boundary of $T$ by

$$
d T\left(f, \pi_{1}, \ldots, \pi_{k-1}\right)=T\left(1, f, \pi_{1}, \ldots, \pi_{k}\right) \quad \forall f d \pi \in \mathcal{E}^{k-1}(X) .
$$

As we noted in the introduction, we use the notation $d T$ instead of the commonly used $\partial T$ because the operator $\partial$ already exists in complex analysis and, together with $\bar{\partial}$, will be defined later on currents.

The functional $d T$ satisfies the first three assumptions, but will not in general be of finite mass; in that case, we say that $T$ is a normal metric current. The space $N_{k}(X)$ of $k$-dimensional normal metric currents is a Banach space when endowed with the norm $\|T\|_{N}=\|T\|+\|d T\|$. 
Definition 2.4 (see Definition 2.4 in [1]). Given a Lipschitz map between complete metric spaces $F: X \rightarrow Y$ and $T \in M_{k}(X)$, we define the pushforward $F_{\sharp} T \in$ $M_{k}(Y)$ of $T$ through $F$ by

$$
F_{\sharp} T\left(f, \pi_{1}, \ldots, \pi_{k}\right)=T\left(f \circ F, \pi_{1} \circ F, \ldots, \pi_{k} \circ F\right) \quad \forall(f, \pi) \in \mathcal{E}^{k}(Y) .
$$

We observe that, if $\mu$ and $\widetilde{\mu}$ are the mass measures of $T$ and $F_{\sharp} T$ respectively, we have

$$
\widetilde{\mu} \leq \operatorname{Lip}(F)^{k} F_{*} \mu .
$$

Moreover, one has $F_{\sharp}(d T)=d\left(F_{\sharp} T\right)$.

Definition 2.5 (see [1], Definition 2.5). Given $T \in M_{k}(X)$ and $\omega=\left(u, v_{1}, \ldots, v_{h}\right)$ an element of $\mathcal{E}^{h}(X)$, with $h \leq k$, the contraction of $T$ with $\omega$ is $T\left\llcorner\omega \in M_{k-h}(X)\right.$ and it is defined by

$$
T\left\llcorner\omega\left(f, \pi_{1}, \ldots, \pi_{k-h}\right)=T\left(f u, v_{1}, \ldots, v_{h}, \pi_{1}, \ldots, \pi_{k-h}\right)\right.
$$

for every $\left(f, \pi_{1}, \ldots, \pi_{k-h}\right) \in \mathcal{E}^{k-h}(X)$.

We have that

$$
\| T\left\llcorner\omega\left\|\leq \sup |u| \prod_{j=1}^{h} \operatorname{Lip}\left(v_{j}\right)\right\| T \| .\right.
$$

Definition 2.6 (see Definition 2.8 in [1]). The support of $T \in M_{k}(X)$ is the least closed set $\operatorname{supp} T$ such that if $\operatorname{supp} f$ does not intersect $\operatorname{supp} T$, then $T(f, \pi)=0$.

By the finiteness of the mass, we can extend a metric current as a functional on $\mathscr{B}^{\infty}(X) \times[\operatorname{Lip}(X)]^{k}$, that is, we can allow the first entry of the metric forms to be a bounded Borel function on $X$. The previous definitions and remarks all extend without changes (see the discussion following Definition 2.6 in [1]).

Proposition 2.7. Let $T \in M_{k}(X)$ be a metric current. Then,

1) $T$ is alternating in $\pi_{1}, \ldots, \pi_{k}$,

2) $T$ satisfies the chain rule and the Leibniz rule,

3) $T=i_{\sharp} S$ with $S \in M_{k}(\operatorname{supp} T)$, and $i: \operatorname{supp} T \rightarrow X$ being the inclusion.

The proofs of these statements can be found in Theorem 3.5 of [1].

We recall a compactness result for normal currents.

Theorem 2.8 (see Theorem 5.2 in [1]). Let $\left(T_{h}\right) \subset N_{k}(X)$ be a bounded sequence and assume that for any integer $p \geq 1$ there exists a compact $K_{p} \subset E$ such that

$$
\left\|T_{h}\right\|\left(E \backslash K_{p}\right)+\left\|d T_{h}\right\|\left(E \backslash K_{p}\right)<\frac{1}{p} \quad \forall h \in \mathbb{N} .
$$

Then, there exists a subsequence $\left(T_{h(n)}\right)$ converging to a current $T \in N_{k}(X)$ such that

$$
\|T\|\left(E \backslash \bigcup_{p=1}^{\infty} K_{p}\right)+\|d T\|\left(E \backslash \bigcup_{p=1}^{\infty} K_{p}\right)=0 .
$$


Finally, we recall the comparison theorem between classical and metric currents, in the form given in Theorem 2 of [10].

Theorem 2.9. Let $U$ be an $N$-dimensional complex manifold, $N \geq 1$, endowed with the distance given by a Hermitian metric. For every $m \geq 0$ there exists an injective linear map $C_{m}: M_{m}(U) \rightarrow \mathscr{D}_{m}(U)$ such that

$$
C_{m}(T)\left(f d g_{1} \wedge \cdots \wedge d g_{m}\right)=T\left(f, g_{1}, \ldots, g_{m}\right)
$$

for all $\left(f, g_{1}, \ldots, g_{m}\right) \in \mathcal{C}_{c}^{\infty}(U) \times\left[\mathcal{C}^{\infty}(U)\right]^{m}$. The following properties hold:

1) for $m \geq 1, d \circ C_{m}=C_{m-1} \circ d$;

2) there exists a positive constant $c_{1}$ such that, for all $T \in M_{m}(U)$,

$$
c_{1}^{-2}\|T\| \leq \mathbf{M}\left(C_{m}(T)\right) \leq c_{1}^{2}\left(\begin{array}{l}
N \\
m
\end{array}\right)\|T\|
$$

3) the restriction of $C_{m}$ to $N_{m}$ is an isomorphism onto $\mathfrak{N}_{m}$;

4) the image of $C_{m}$ contains the space $\mathfrak{F}_{m}(U)$.

\subsection{Rectifiable currents and slicing}

Let $\mathcal{H}^{k}$ be the $k$-dimensional Hausdorff measure on $X$.

Definition 2.10 (see Definition 5.3 in [2]). We say that a $\mathcal{H}^{k}$-measurable set $S \subset X$ is countably $\mathcal{H}^{k}$-rectifiable if there exist sets $A_{i} \subset \mathbb{R}^{k}$ and Lipschitz functions $f_{i}: A_{i} \rightarrow X$ such that

$$
\mathcal{H}^{k}\left(S \backslash \bigcup_{i=1}^{\infty} f_{i}\left(A_{i}\right)\right)=0 .
$$

Lemma 2.11 (see Lemma 4.1 in [1]). Let $S \subset X$ be countably $\mathcal{H}^{k}$-rectifiable. Then, there exist finitely or countably many compact sets $K_{i} \subset \mathbb{R}^{k}$ and bi-Lipschitz maps $f_{i}: K_{i} \rightarrow S$ such that their images are pairwise disjoint and $\mathcal{H}^{k}\left(S \backslash \cup_{i} f_{i}\left(K_{i}\right)\right)=0$.

Definition 2.12 (see Definition 4.2 in [1]). A current $T \in M_{k}(X)$ is said to be rectifiable if

1) $\|T\|$ is concentrated on a countably $\mathcal{H}^{k}$-rectifiable set;

2) $\|T\|$ vanishes on $\mathcal{H}^{k}$-negligible Borel sets.

The space of rectifiable currents is denoted by $\mathcal{R}_{k}(X)$.

We say that a rectifiable current $T$ is integer rectifiable if for any $\phi \in \operatorname{Lip}\left(X, \mathbb{R}^{k}\right)$ and open set $B$ in $X$ one has $\phi_{\sharp}\left(T\llcorner B)=[u]\right.$ with $u \in L^{1}\left(\mathbb{R}^{k}, \mathbb{Z}\right)$. The space of such currents is denoted by $\mathcal{I}_{k}(X)$.

We remark that in some of the standard references for geometric measure theory the terminology rectifiable is used to denote what here we call integer rectifiable.

We define the spaces of integral currents as follows:

$$
I_{k}(X)=\mathcal{I}_{k} \cap N_{k}(X) .
$$


Theorem 2.13 (see Theorem 4.5 in [1]). Let $T \in M_{k}(X), k \geq 1$. Then $T \in \mathcal{R}_{k}(X)$ (resp. $\left.T \in \mathcal{I}_{k}(X)\right)$ if and only if there exist a sequence $\left\{K_{i}\right\}$ of compact sets in $\mathbb{R}^{k}$, a sequence $\left\{\theta_{i}\right\}$ of functions in $L^{1}\left(\mathbb{R}^{k}, \mathbb{R}\right)\left(\right.$ resp. $\left.L^{1}\left(\mathbb{R}^{k}, \mathbb{Z}\right)\right)$ with $\operatorname{supp} \theta_{i} \subset K_{i}$, and a sequence $\left\{f_{i}\right\}$ of bi-Lipschitz maps $f_{i}: K_{i} \rightarrow X$ such that

$$
\|T\|(A)=\sum_{i}\left\|\left(f_{i}\right)_{\sharp}\left[\theta_{i}\right]\right\|(A) \quad \text { and } \quad T(f, \pi)=\sum_{i}\left(f_{i}\right)_{\sharp}\left[\theta_{i}\right](f, \pi)
$$

for every Borel set $A \subset X$ and for every $(f, \pi) \in \mathcal{E}^{k}(X)$.

Definition 2.14 (see Definition 4.7 in [1]). Given $T \in \mathcal{R}_{k}(X)$, we set

$$
S_{T}=\left\{x \in X: \Theta_{k}(\|T\|, x)>0\right\}
$$

and we define the size of $T$ as

$$
\mathbf{S}(T)=\mathcal{H}^{k}\left(S_{T}\right)
$$

One can show that $S_{T}$ is countably $\mathcal{H}^{k}$-rectifiable, that $\|T\|$ is concentrated on $S_{T}$ and that any other Borel set on which $\|T\|$ is concentrated includes $S_{T}$ up to $\mathcal{H}^{k}$-negligible sets.

Given $T \in M_{k}(X), \pi \in \operatorname{Lip}\left(X, \mathbb{R}^{m}\right)$, we define the slice $\langle T, \pi, x\rangle \in M_{k-m}(X)$ by

$$
\langle T, \pi, x\rangle(f, \eta)=\lim _{\epsilon \rightarrow 0} T\left(f \rho_{\epsilon} \circ \pi, \pi, \eta\right)
$$

where $\rho_{\epsilon}$ is any family of mollifiers, for every $x \in \mathbb{R}^{m}$ for which the limit exists. This definition differs from the one given in [1], page 31 , but it is shown to be equivalent to it in the proof of Theorem 5.6 in [1]. The following result is not present in [1] as it is stated here, but can be deduced from Theorem 5.6 there.

Theorem 2.15. If $T \in N_{k}(X)$ and $\pi \in \operatorname{Lip}\left(X, \mathbb{R}^{m}\right)$, then

1) for $\mathcal{L}^{m}$-almost every $x \in \mathbb{R}^{m}$, the slice $\langle T, \pi, x\rangle$ exists and is normal and $d\langle T, \pi, x\rangle=(-1)^{m}\langle d T, \pi, x\rangle ;$

2) for all $(f, g) \in \mathcal{B}^{\infty} \times[\operatorname{Lip}(X)]^{k-m}$,

$$
\int_{\mathbb{R}^{m}}\langle T, \pi, x\rangle(f, g) d x=T\llcorner(1, \pi)(f, g) ;
$$

3) for every $\| T\llcorner(1, \pi) \|$-measurable set $B \subset X$,

$$
\int_{\mathbb{R}^{m}}\|\langle T, \pi, x\rangle\|(B) d x=\| T\llcorner(1, \pi) \|(B) .
$$




\subsection{Rectifiable currents in Banach spaces}

Let us specialize the theory of metric currents to the case when $X$ is a Banach space $(E,\|\cdot\|)$. We recall some notions on metric differentiability, Jacobians, area and coarea formulas from [1] and [2].

Let us suppose that $E$ is a $w^{*}$-separable dual space, i.e., that $E=G^{*}$ and that there exists a sequence $\left(\phi_{h}\right)_{h} \subset \operatorname{Lip}_{1}(G)$ such that

$$
\|x-y\|_{G}=\sup _{h}\left|\phi_{h}(x)-\phi_{h}(y)\right| \quad \forall x, y \in G .
$$

Given instead $x, y \in E$, we define

$$
d_{w}(x, y)=\sum_{n=0}^{\infty} 2^{n}\left|\left\langle x-y, g_{n}\right\rangle\right|
$$

where $\left\{g_{n}\right\}_{n}$ is a dense subset of the unit ball of $G$. The topology induced by $d_{w}$ on the bounded sets of $E$ is called $w^{*}$-topology.

Definition 2.16 (see Definition 6.1 in [1]). A sequence $\left(T_{h}\right) \subset M_{k}(E)$ is said to $w^{*}$-converge to $T \in M_{k}(E)$ if $T_{h}(f d \pi)$ tends to $T(f d \pi)$ for every $f d \pi \in \mathcal{E}^{k}(E)$ with $w^{*}$-continuous coefficients.

We have the following weak*-compactness result.

Theorem 2.17 (see Theorem 6.6 in [1]). Let $Y$ be a $w^{*}$-separable dual space, let $\left(T_{h}\right) \subset N_{k}(Y)$ be a bounded sequence, and assume that for any $\epsilon>0$ there exists $R>0$ such that $K_{h}=\bar{B}_{R}(0) \cap \operatorname{supp} T_{h}$ are equicompact and

$$
\sup _{h \in \mathbb{N}}\left\|T_{h}\right\|\left(Y \backslash K_{h}\right)+\left\|d T_{h}\right\|\left(Y \backslash K_{h}\right)<\epsilon .
$$

Then, there exists a subsequence $\left(T_{h(n)}\right) w^{*}$-converging to $T \in N_{k}(Y)$. Moreover, $T$ has compact support if $\operatorname{supp} T_{h}$ are equibounded.

Definition 2.18 (see Definition 3.1 in [2]). We say that $f: \mathbb{R}^{k} \rightarrow E$ is metrically differentiable at $x \in \mathbb{R}^{k}$ if there exists a seminorm $\|\cdot\|_{x}$ in $\mathbb{R}^{k}$ such that

$$
\|f(x)-f(y)\|_{E}-\|x-y\|_{x}=o(|x-y|) .
$$

This seminorm will be said to be the metric differential of $f$ at $x$ and denoted by $m d f(x)$.

Definition 2.19 (see Definition 3.4 in [1]). We say that $f: \mathbb{R}^{k} \rightarrow E$ is $w^{*}$-differentiable at $x$ if there exists a linear map $L: \mathbb{R}^{k} \rightarrow E$ satisfying

$$
w^{*}-\lim _{y \rightarrow x} \frac{f(x)-f(y)-L(y-x)}{|x-y|}=0 .
$$

This map $L$ will be said to be the $w^{*}$-differential of $f$ at $x$ and denoted by $w d f_{x}$. 
Theorem 2.20 (see Theorem 3.5 in [2]). For a Lipschitz map $f: \mathbb{R}^{k} \rightarrow E$, we have

$$
m d f_{x}(v)=\left\|w d f_{x}(v)\right\|
$$

for $\mathcal{H}^{k}$-a.e. $x \in \mathbb{R}^{k}$, for every $v \in \mathbb{R}^{k}$.

Definition 2.21 (see Definition 5.5 in [2]). Given a countably $\mathcal{H}^{k}$-rectifiable set $S$ in $E$, if $f_{i}$ and $A_{i}$ are as in Definition 2.10, the approximate tangent space to $S$ in $f_{i}(x)$ is

$$
\operatorname{Tan}^{(k)}\left(S, f_{i}(x)\right)=w d\left(f_{i}\right)_{x}\left(\mathbb{R}^{k}\right)
$$

This definition makes sense for $\mathcal{H}^{k}$-a.e. $x \in A_{i}$ and it is well posed as the dimension of the tangent is $\mathcal{H}^{k}$-a.e. $k$ and it does not depend on the $f_{i}$ 's; moreover

$$
\operatorname{Tan}^{(k)}\left(S_{1}, y\right)=\operatorname{Tan}^{(k)}\left(S_{2}, y\right)
$$

for $\mathcal{H}^{k}$-a.e. $y \in S_{1} \cap S_{2}$.

Definition 2.22 (see Definition 4.1 in [2]). Let $L: \mathbb{R}^{k} \rightarrow E$ be linear. The $k$-Jacobian of $L$ is defined by

$$
\mathbf{J}_{k}(L)=\frac{\omega_{k}}{\mathcal{H}^{k}(\{x:\|L(x)\| \leq 1\})}=\frac{\mathcal{H}^{k}\left(\left\{L(x): x \in B_{1}\right\}\right)}{\omega_{k}},
$$

where $B_{1}$ is the unit ball of $\mathbb{R}^{k}$ and $\omega_{k}=\mathcal{H}^{k}\left(B_{1}\right)$.

The $k$-Jacobian $\mathbf{J}_{k}$ satisfies the product rule for Jacobians:

$$
\mathbf{J}_{k}(L \circ M)=\mathbf{J}_{k}(L) \mathbf{J}_{k}(M)
$$

In the same way, we can define the $k$-Jacobian of a seminorm $s$ on $\mathbb{R}^{k}$ :

$$
\mathbf{J}_{k}(s)=\frac{\omega_{k}}{\mathcal{H}^{k}(\{x: s(x) \leq 1\})} .
$$

Theorem 2.23 (see Theorem 5.1 in [2]). Let $f: \mathbb{R}^{k} \rightarrow E$ be a Lipschitz function. Then

$$
\int_{\mathbb{R}^{k}} \theta(x) \mathbf{J}_{k}\left(m d f_{x}\right) d x=\int_{E} \sum_{x \in f^{-1}(y)} \theta(x) d \mathcal{H}^{k}(y)
$$

for any Borel function $\theta: \mathbb{R}^{k} \rightarrow[0,+\infty]$, and

$$
\int_{A} \theta(f(x)) \mathbf{J}_{k}\left(m d f_{x}\right) d x=\int_{E} \theta(y) \mathcal{H}^{0}\left(A \cap f^{-1}(y)\right) d \mathcal{H}^{k}(y)
$$

for $A$ a Borel set in $\mathbb{R}^{k}$ and $\theta: E \rightarrow[0,+\infty]$ a Borel function.

Moreover, we can also define the tangential differential on a rectifiable set. 
Theorem 2.24 (see Theorem 8.1 in [2]). Let $Y$ and $Z$ be duals of separable Banach spaces and let $S \subset Y$ be countably $\mathcal{H}^{k}$-rectifiable and let $g \in \operatorname{Lip}(S, Z)$. Let $\theta: S \rightarrow$ $(0,+\infty)$ be integrable with respect to $\mathcal{H}^{k}\left\llcorner S\right.$ and set $\mu=\theta \mathcal{H}^{k}\llcorner S$.

Then, for $\mathcal{H}^{k}$-a.e. $x \in S$, there exist a linear and $w^{*}$-continuous map $L: Y \rightarrow Z$ and a Borel se $S^{x} \subset S$ such that $\Theta_{k}^{*}\left(\mu\left\llcorner S^{x}, x\right)=0\right.$ (the upper $k$-dimensional density of $\mu\left\llcorner S^{x}\right.$ in $\left.x\right)$ and

$$
\lim _{S \backslash S^{x} \ni y \rightarrow x} \frac{d_{w}(g(y), g(x)+L(y-x))}{|y-x|}=0 .
$$

The map $L$ is uniquely determined on $\operatorname{Tan}^{(k)}(S, x)$ and its restriction to this space, denoted by $d^{S} g_{x}$, satisfies the chain rule

$$
w d(g \circ h)_{y}=d^{S} g_{h(y)} \circ w d h_{y} \text { for } \mathfrak{L}^{k}-\text { a.e. } y \in A
$$

for any Lipschitz function $h: A \rightarrow S, A \subset \mathbb{R}^{k}$.

We have therefore a general area formula.

Theorem 2.25 (see Theorem 8.2 in [2]). Let $g: E \rightarrow F$ be a Lipschitz function between Banach spaces and let $S \subset E$ be a countably $\mathcal{H}^{k}$-rectifiable set. Then

$$
\int_{S} \theta(x) \mathbf{J}_{k}\left(d^{S} g_{x}\right) d \mathcal{H}^{k}(x)=\int_{F} \sum_{x \in S \cap g^{-1}(y)} \theta(x) d \mathcal{H}^{k}(y)
$$

for any Borel function $\theta: S \rightarrow[0,+\infty]$, and

$$
\int_{A} \theta(g(x)) \mathbf{J}_{k}\left(d^{S} g_{x}\right) d \mathcal{H}^{k}(x)=\int_{F} \theta(y) \mathcal{H}^{0}\left(A \cap g^{-1}(y)\right) d \mathcal{H}^{k}(y)
$$

for any Borel set $A \subset E$ and any Borel function $\theta: F \rightarrow[0,+\infty]$.

Finally, we mention a compactness theorem proved in [3].

Theorem 2.26 (see Theorem 1.4 in [3]). Consider a normed space E such that $E^{*}$ is separable, $n \in \mathbb{N}$, and a sequence $\left(T_{h}\right) \subset N_{k}\left(E^{*}\right)$ such that we have

$$
M=\sup _{h} M\left(T_{h}\right)<+\infty \quad \text { and } \quad M_{d}=\sup _{h} M\left(d T_{h}\right)<+\infty
$$

and the $w^{*}$-tightness condition

$$
\lim _{R \rightarrow \infty} \sup _{h}\left[\left\|T_{h}\right\|\left(E^{*} \backslash B_{R}(0)\right)+\left\|d T_{h}\right\|\left(E^{*} \backslash B_{R}(0)\right)\right]=0 .
$$

Then there exists a subsequence $\left(T_{h(n)}\right)_{n} w^{*}$-converging to $T \in N_{k}\left(E^{*}\right)$ with $M(T) \leq$ $M$ and $M(d T) \leq M_{d}$. 


\section{Metric currents on complex Banach spaces}

The results collected here and in the next subsection appear in [10], where we refer to for detailed proofs.

We examine the behavior of metric currents in relation with their projections on finite dimensional subspaces. In order to recover informations on the whole space from its finite dimensional subspaces, we introduce the following category of Banach spaces (see also [13]).

A (complex) Banach space $E$ is said to have the projective approximation property (PAP for short) if there exist a constant $a$ and an increasing collection $\left\{E_{t}\right\}_{t \in T}$ of finite dimensional subspaces of $E$ such that

1) $\left\{E_{t}\right\}_{t \in I}$ is a directed set for the inclusion;

2) $E=\overline{\bigcup_{t \in I} E_{t}}$;

3) for every $t \in I$ there exists a projection $p_{t}: E \rightarrow E_{t}$ with $\left\|p_{t}\right\| \leq a$.

Every Banach space with a Schauder basis has the PAP. Two important cases of PAP Banach spaces with no Schauder basis are $\mathcal{C}(K)$, the space of continuous functions on a compact space $\mathrm{K}$ with the sup norm and $L^{p}(X, \mu)$, with $1 \leq p \leq$ $+\infty$, where $X$ is a locally compact space and $\mu$ being a positive Radon measure. In this section, we will work with Banach spaces having the PAP; we will endow the set $I$ of indices with the partial ordering coming from the inclusion relation between subspaces.

Proposition 3.1. Let $f \in \operatorname{Lip}(E)$ and define $f_{t}=f \circ p_{t}$. Then $f_{t} \rightarrow f$ pointwise and $\operatorname{Lip}\left(f_{t}\right) \leq a \operatorname{Lip}(f)$, for every $t \in I$.

Proposition 3.2. Let $T \in M_{k}(E)$ and define $T_{t}=\left(\pi_{t}\right)_{\sharp}(T) \in M_{k}\left(E_{t}\right)$ for every $t \in I$ such that $\operatorname{dim}_{\mathbb{C}} E_{t} \geq k$. By means of the inclusion $i_{t}: E_{t} \rightarrow E$, we can consider $T_{t}$ as an element of $M_{k}(E)$ and then, $T_{t} \rightarrow T$ weakly.

Let $\left\{E_{t}, p_{t}\right\}_{t \in I}$ be the countable collection of subspaces and projections given by PAP. We call it a projective approximating sequence (PAS for short) if $p_{t} \circ p_{s}=$ $p_{\min \{s, t\}}$.

We note that every separable Hilbert space or, more generally, every Banach space with a Schauder basis contains a PAS.

Theorem 3.3. Let us suppose that $\left\{E_{t}, p_{t}\right\}$ is a PAS in E. If we are given a collection of metric currents $\left\{T_{t}\right\}_{t \in I}$ such that

1) $T_{t} \in N_{k}\left(E_{t}\right)$,

2) $\left(\left.p_{t}\right|_{E_{t^{\prime}}}\right)_{\sharp} T_{t^{\prime}}=T_{t}$ for every $t, t^{\prime} \in I$ with $t^{\prime}>t$,

3) $\left\|T_{t}\right\| \leq\left(p_{t}\right)_{*} \mu$ and $\left\|d T_{t}\right\| \leq\left(p_{t}\right)_{*} \nu$ for every $t \in I$ and some $\mu, \nu$ finite Radon measures on $E$.

Then there exists $T \in N_{k}(E)$ such that $\left(p_{t}\right)_{\sharp} T=T_{t}$ for every $t \in I$. 
We can substitute the request of the existence of a PAS and the compatibility condition (hypothesis 2)) with an assumption on the existence of a global object. A metric functional is a function $T: \mathcal{E}^{k}(E) \rightarrow \mathbb{C}$ which is subadditive and positively 1-homogeneous with respect to every variable. For metric functionals, we can define mass, boundary and pushforward (see Section 2 of [1]).

Proposition 3.4. Let $E$ be a Banach space with PAP. Suppose that $T: \mathcal{E}^{k}(E) \rightarrow \mathbb{C}$ is a metric functional with $T$ and $\mathrm{d} T$ of finite mass, such that $\left(p_{t}\right)_{\sharp} T \in N_{k}\left(E_{t}\right)$ for every $t \in I$. Then $T \in N_{k}(E)$.

\subsection{Bidimension}

We say that $T \in M_{m}(U)$ is of bidimension $(p, q)$ if

$$
T\left(f, \pi_{1}, \ldots, \pi_{m}\right)=0
$$

whenever there exists $I \subset\{1, \ldots, m\}$, with $|I|>p$, such that $\left.\pi_{i}\right|_{\operatorname{supp}(f)}$ is holomorphic for every $i \in I$, or $J \subset\{1, \ldots, m\}$, with $|J|>q$, such that $\left.\pi_{i}\right|_{\operatorname{supp}(f)}$ is antiholomorphic for every $j \in J$. For a careful analysis of the notion of holomorphy in this context we refer the interested reader to the first chapters in [13]. Here we only notice that Lipschitz holomorphic functions are not necessarily dense in the space of Lipschitz functions.

However, inspired by the links we found between the finite dimensional projections of a current and the current itself, we would like to give a different characterization of $(p, q)$-currents.

We say that $T \in M_{k}(E)$ is finitely of bidimension $(p, q)$ if every finite dimensional projection of it is a $(p, q)$-current.

Proposition 3.5. Let $E$ be a Banach space with PAP. Then $T \in M_{k}(E)$ is a $(p, q)$-current if and only if it is finitely so.

In what follows, we will denote by $M_{p, q}(E)$ the space of currents $T \in M_{k}(X)$ with $k=p+q$ which are of bidimension $(p, q)$. Given a generic element $T \in M_{k}(E)$, a Dolbeautl decomposition for $T$ is a finite collection of currents $S_{j} \in M_{p_{j}, q_{j}}(E)$ such that $p_{j}+q_{j}=k$ for every $j,\left(p_{j}, q_{j}\right) \neq\left(p_{k}, q_{k}\right)$ if $j \neq k$ and

$$
T=\sum_{j} S_{j} .
$$

The current $S_{j}$ will be called $\left(p_{j}, q_{j}\right)$-component of $T$ and will also be denoted by $T_{p_{j}, q_{j}}$.

As an application of Theorem 3.3, we have the following result about the existence of a Dolbeault decomposition for $T \in M_{k}(E)$.

Proposition 3.6. Let us suppose that $\left\{E_{t}, p_{t}\right\}$ is a PAS in E. Let $T \in N_{k}(E)$; if $T_{t}$ has a Dolbeault decomposition in normal $(p, q)$-currents in $E_{t}$ for all $t \in T$, with a finite Radon measure $\nu$ (independent of $t$ ) whose pushforward dominates the boundaries' masses, then also T admits a Dolbeault decomposition. 
Remark 3.7. In general it is not easy to verify the hypotheses of Proposition 3.6 for a current $T \in N_{k}(E)$; however, this result is an example of a general phenomenon: in a Banach space with the projective approximation property, it is often enough to check a certain property for finite dimensional subspaces in order to obtain that it holds for the whole space. For instance, any equality between currents holds in $E$ if and only if it holds finitely, namely whenever the currents are pushed forward through a finite rank projection.

Employing the idea given in this Remark, we can show the following result of uniqueness, which shows that it is meaningful to speak of the Dolbeaut decomposition and the $(p, q)$-components of $T$.

Corollary 3.8. If $E$ is a Banach space with $P A P$ and $T \in M_{k}(E)$ admits a Dolbeault decomposition, then it is unique.

A metric current is not always decomposable in $(p, q)$-components (see Examples 2 and 3 in [10]), but it is always possible to obtain a (unique) decomposition in terms of metric functionals which satisfy requests 1), 3), and 4) in Definition 2.1. We refer to [10] for further discussions. We will denote these components as well by $T_{p, q}$, because, as soon as they are metric currents, they automatically are the $(p, q)$-components of $T$, but we will reserve the expression Dolbeault decomposition for a decomposition in metric currents.

Let us suppose that $T$ is a $(p, q)$-current whose boundary admits a Dolbeault decomposition. Then we can define $\partial T$ and $\bar{\partial} T$ as follows.

Write $d T=S_{1}+\cdots+S_{h}$ with $S_{i} \in M_{p_{i}, q_{i}}(U)$ where $p_{i}+q_{i}=p+q-1=m$ since $d T \in M_{p+q-1}(U)$. If $(f, \pi)$ is a $m$-form of pure type $\left(p_{i}, q_{i}\right)$, then

$$
S_{p_{i}, q_{i}}(f, \pi)=d T(f, \pi)=T(1, f, \pi)
$$

if $p_{i}>p$ or $q_{i}>q$, and consequently $T(1, f, \pi)=0$, since $T$ is a $(p, q)$-current. Therefore, we can only have two cases: $p=p_{i}$ and $q-1=q_{i}$, or $p-1=p_{i}$ and $q=q_{i}$, i.e.,

$$
d T=S_{p, q-1}+S_{p-1, q},
$$

and we put

$$
\partial T=S_{p-1, q} \quad \text { and } \quad \bar{\partial} T=S_{p, q-1} .
$$

Therefore, if a current $T$ admits a decomposition in $(p, q)$ components, we can define $\partial T$ and $\bar{\partial} T$ setting

$$
\partial T=\sum_{i=1}^{h} \partial T_{i} \quad \text { and } \quad \bar{\partial} T=\sum_{i=1}^{h} \bar{\partial} T_{i},
$$

where $T=T_{1}+\cdots+T_{h}$ is the $(p, q)$ decomposition.

Proposition 3.9. If $H: E \rightarrow F$ is a holomorphic map between complex Banach spaces, then, for every current $T \in M_{m}(E)$ for which $\partial T$ and $\bar{\partial} T$ are defined, the following hold:

$$
H_{\sharp} \partial T=\partial H_{\sharp} T \quad \text { and } \quad H_{\sharp} \bar{\partial} T=\bar{\partial} H_{\sharp} T .
$$


Moreover, it is easy to check that $C_{m} \circ \partial=\partial \circ C_{m}$ and $C_{m} \circ \bar{\partial}=\bar{\partial} \circ C_{m}$, where $C_{m}$ is the map given by Theorem 2.9 .

Proposition 3.10. We have that $\partial^{2}=\bar{\partial}^{2}=0$ and $\partial \bar{\partial}=-\bar{\partial} \partial$.

\section{2. $\bar{\partial}$ and slicing}

Let $T$ be a normal metric current whose boundary admits a Dolbeault decomposition. Then supp $\bar{\partial} T$, supp $\partial T \subseteq \operatorname{supp} d T$ and, moreover, $\|\bar{\partial} T\|_{A},\|\partial T\|_{A} \leq C\|d T\|_{A}$ for every $A \subset X$. In particular, if $d T$ is rectifiable, then $\bar{\partial} T$ and $\partial T$ are too.

The slices of a current $T$ through a map $\pi: E \rightarrow \mathbb{R}^{n}$ are defined by

$$
\langle T, \pi, x\rangle(f, \eta)=\lim _{\epsilon \rightarrow 0} T\left(\rho_{\epsilon, x} f, \pi, \eta\right)=\lim _{\epsilon \rightarrow 0}(-1)^{n(k-1-n)} \pi_{\sharp}\left(T\llcorner(f, \eta))\left(\rho_{\epsilon, x}, x_{1}, \ldots, x_{n}\right)\right.
$$

with $\rho_{\epsilon, x}$ any family of smooth approximations of $\delta_{x}$. If $\pi: E \rightarrow \mathbb{R}^{2 n} \cong \mathbb{C}^{n}$, we can write the slices as

$$
\langle T, \pi, x\rangle=\lim _{\epsilon \rightarrow 0} \pi_{\sharp}\left(T\llcorner(f, \eta))\left(\rho_{\epsilon, x}, z_{1}, \bar{z}_{1}, \ldots, z_{n}, \bar{z}_{n}\right) .\right.
$$

Proposition 3.11. The operators $\partial$ and $\bar{\partial}$ commute with the slicing through holomorphic maps.

Proof. Let $T \in N_{k}(E)$ be a normal current, such that $\partial T$ and $\bar{\partial} T$ are again normal. Let $\pi: E \rightarrow \mathbb{C}^{n}$ be a holomorphic map.

We start with the finite dimensional case, supposing that $E=\mathbb{C}^{N}$.

Let $(f, \eta)$ be a $(k-2 n-1)$-metric form with $\mathcal{C}^{2}$ coefficients. We treat only the case of $\bar{\partial} T$, the proof for $\partial T$ being analogous.

Let $z_{1}, \ldots, z_{n}$ and $w_{1}, \ldots, w_{N}$ be holomorphic coordinates in $\mathbb{C}^{n}$ and $\mathbb{C}^{N}$, respectively. The slice $\langle\bar{\partial} T, \pi, x\rangle$ exists for a.e. $x$, by Theorem 2.15 , and we have

$$
\langle\bar{\partial} T, \pi, x\rangle(f, \eta)=\lim _{\epsilon \rightarrow 0} \pi_{\sharp}\left((\bar{\partial} T)\llcorner(f, \eta))\left(\rho_{\epsilon, x}, z_{1}, \bar{z}_{1}, \ldots, z_{n}, \bar{z}_{n}\right) .\right.
$$

Now, we set $\widetilde{\eta}^{j h}$ to be the $(k-2 n-1)$-tuple differing from $\eta$ only in the $h$-th component, which is $(-1)^{h} \partial \eta_{h} / \partial \bar{w}_{j}$. By Proposition 6 in [10],

$$
(\bar{\partial} T)\left\llcorner(f, \eta)=(-1)^{k-1} \bar{\partial}\left(T\llcorner(f, \eta))+\sum_{j=1}^{N} T\left\llcorner\left(\partial f / \partial \bar{w}_{j}, \bar{w}_{j}, \eta\right)+\sum_{h, j} T\left\llcorner\left(f, \bar{w}_{j}, \widetilde{\eta}^{j h}\right),\right.\right.\right.\right.
$$

and we note that $T\llcorner(f, \eta)$ is a $2 n+1$-form, so

$$
\pi_{\sharp} \bar{\partial} T\left\llcorner(f, \eta)=\bar{\partial} \pi_{\sharp} T\llcorner(f, \eta)=0\right.
$$


by Proposition 3.9, as $\pi$ is holomorphic. It follows that

$$
\begin{aligned}
& \pi_{\sharp}\left((\bar{\partial} T)\llcorner(f, \eta))\left(\rho_{\epsilon, x}, z_{1}, \ldots, \bar{z}_{n}\right)=\sum_{j=1}^{N} \pi_{\sharp}\left(T\left\llcorner\left(\partial f / \partial \bar{w}_{j}, \bar{w}_{j}, \eta\right)\right)\left(\rho_{\epsilon, x}, z_{1}, \ldots, \bar{z}_{n}\right)\right.\right. \\
& \quad+\sum_{j, h} \pi_{\sharp}\left(T\left\llcorner\left(f, \bar{w}_{j}, \widetilde{\eta}^{j h}\right)\right)\left(\rho_{\epsilon, x}, z_{1}, \ldots, \bar{z}_{n}\right)\right. \\
&=\sum_{j=1}^{N} T\left(\frac{\partial f}{\partial \bar{w}_{j}} \cdot\left(\rho_{\epsilon, x} \circ \pi\right), \pi_{1}, \bar{\pi}_{1}, \ldots, \pi_{n}, \bar{\pi}_{n}, \eta\right) \\
&+\sum_{j, h} T\left(f \cdot\left(\rho_{\epsilon, x} \circ \pi\right), \pi_{1}, \ldots, \bar{\pi}_{n}, \widetilde{\eta}^{j h}\right) \\
&= \sum_{j=1}^{N} T\left\llcorner\left(\rho_{\epsilon, x} \circ \pi, \pi_{1}, \ldots, \bar{\pi}_{n}\right)\left(\partial f / \partial \bar{w}_{j}, \bar{w}_{j}, \eta\right)\right. \\
&+\sum_{j, h} T\left\llcorner\left(\rho_{\epsilon, x} \circ \pi, \pi_{1}, \ldots, \bar{\pi}_{n}\right)\left(f, \bar{w}_{j}, \widetilde{\eta}^{j h}\right) .\right.
\end{aligned}
$$

Now, again by Lemma 1 in [10], we have

$$
\begin{aligned}
\bar{\partial}\langle T, \pi, x\rangle(f, \eta)= & \sum_{j=1}^{N}\langle T, \pi, x\rangle\left(\frac{\partial f}{\partial \bar{w}_{j}}, \bar{w}_{j}, \eta\right)+\sum_{j, h}\langle T, \pi, x\rangle\left(f, \bar{w}_{j}, \widetilde{\eta}^{j h}\right) \\
= & \sum_{j=1}^{N} \lim _{\epsilon \rightarrow 0} T\left\llcorner\left(\rho_{\epsilon, x} \circ \pi, \pi_{1}, \ldots, \bar{\pi}_{n}\right)\left(\frac{\partial f}{\partial \bar{w}_{j}}, \bar{w}_{j}, \eta\right)\right. \\
& +\sum_{j, h} \lim _{\epsilon \rightarrow 0} T\left\llcorner\left(\rho_{\epsilon, x} \circ \pi, \pi_{1}, \ldots, \bar{\pi}_{n}\right)\left(f, \bar{w}_{j}, \widetilde{\eta}^{j h}\right),\right.
\end{aligned}
$$

and by the previous computation this is equal to

$$
\lim _{\epsilon \rightarrow 0} \pi_{\sharp}\left((\bar{\partial} T)\llcorner(f, \eta))\left(\rho_{\epsilon, x}, \pi_{1}, \ldots, \bar{\pi}_{n}\right)=\langle\bar{\partial} T, \pi, x\rangle(f, \eta) .\right.
$$

Therefore, for every $(f, \eta)$ with $f \in \mathcal{C}^{2}$,

$$
\langle\bar{\partial} T, \pi, x\rangle(f, \eta)=\bar{\partial}\langle T, \pi, x\rangle(f, \eta) .
$$

This means that the functional $\bar{\partial}\langle T, \pi, x\rangle$ can be extended to all the metric forms as a metric current, by defining it equal to $\langle\bar{\partial} T, \pi, x\rangle$.

If $E$ is infinite-dimensional, the previous argument gives that

$$
\langle\bar{\partial} T, \pi, x\rangle(f, \eta)=\bar{\partial}\langle T, \pi, x\rangle(f, \eta)
$$

for every metric form whose coefficients depend on a finite number of variables. This implies that

$$
\left(p_{t}\right)_{\sharp}\langle\bar{\partial} T, \pi, x\rangle=\left(p_{t}\right)_{\sharp} \bar{\partial}\langle T, \pi, x\rangle(f, \eta)
$$

for every finite rank projection $p_{t}$, and consequently that

$$
\langle\bar{\partial} T, \pi, x\rangle=\bar{\partial}\langle T, \pi, x\rangle .
$$


As already said, the previous proof works also for the $\partial$ :

$$
\partial\langle T, \pi, x\rangle=\langle\partial T, \pi, x\rangle .
$$

Moreover, if $\partial \bar{\partial} T$ is a metric current, then

$$
\partial \bar{\partial}\langle T, \pi, x\rangle=\langle\partial \bar{\partial} T, \pi, x\rangle,
$$

or, which is the same,

$$
d d^{c}\langle T, \pi, x\rangle=\left\langle d d^{c} T, \pi, x\right\rangle .
$$

\section{Analytic sets in Hilbert spaces}

There are many possible definitions for a finite dimensional analytic set in an infinite-dimensional space. Here we adopt the following (see [14]).

Definition 4.1. Let $H$ be a complex Hilbert space (or more generally a Banach space). A closed set $A \subset H$ will be called a finite-dimensional analytic set in $H$ if, locally in $H, A$ is an analytic subspace of a complex submanifold of $H$ of finite dimension.

Remark 4.2. This definition is equivalent to the one given by Douady in [5] (see [14] for the details).

An equivalent definition is the following (see [4]): $A \subset H$ is a finite-dimensional analytic set if for each $v \in H$ there exist a neighborhood $U$, another complex Hilbert space $H^{\prime}$ and a holomorphic map $F: U \rightarrow H^{\prime}$ whose differential has finite dimensional kernel such that $A \cap U=F^{-1}(0)$. This follows easily from the implicit function theorem; this turns out to be equivalent to asking for a map whose differential has finite dimensional kernel and cokernel (i.e., a Fredholm map) such that $A \cap U=F^{-1}(0)$.

We recall a result from [14].

Theorem 4.3. Let $X$ be an analytic subset of finite dimension, $W$ be a hilbertian manifold and $f: X \rightarrow W$ a proper holomorphic map. Then, $f(X)$ is a finitedimensional analytic subset of $W$.

Example 4.4. The properness assumption cannot be dropped as shown by the following example. Let us consider the space $\ell^{2}$ of square-summable sequences of complex numbers and consider the holomorphic map $g: \mathbb{D}^{2} \rightarrow \ell^{2}$ given by

$$
g(z, w)=\left\{z w^{n}\right\}_{n \in \mathbb{N}} .
$$

The preimage of $\{0\}$ is $\{z=0\}$, which is not compact. Let $X=g\left(\mathbb{D}^{2}\right)$ and assume, by a contradiction, that there exists a holomorphic function $\Phi: \ell^{2} \rightarrow \mathbb{C}$ is a holomorphic function vanishing on $X$; then $\Phi \circ g=0$, and consequently,

$$
0=\Phi \circ g(z, w)=\sum_{n, m \geq 0} \frac{\partial^{n+m} \Phi \circ g}{\partial z^{n} \partial w^{m}}(0,0) \frac{z^{n} w^{m}}{n ! m !} .
$$


One has

$$
\begin{aligned}
0 & =\frac{\partial \Phi \circ g}{\partial z}(0,0)=\frac{\partial \Phi}{\partial e_{0}}(0) \\
0 & =\frac{\partial^{2} \Phi \circ g}{\partial w \partial z}(0,0)=\frac{\partial}{\partial w}\left(\sum_{j} \frac{\partial \Phi}{\partial e_{j}}(g) \frac{\partial g_{j}}{\partial z}\right)(0,0) \\
& =\left(\sum_{j, k} \frac{\partial^{2} \Phi}{\partial e_{k} \partial e_{j}}(g) \frac{\partial g_{k}}{\partial w} \frac{\partial g_{j}}{\partial z}+\sum_{j} \frac{\partial \Phi}{\partial e_{j}} \frac{\partial^{2} g_{j}}{\partial w \partial z}\right)(0,0)=\frac{\partial \Phi}{\partial e_{1}}(0,0),
\end{aligned}
$$

since, for all $k$,

$$
\frac{\partial g_{k}}{\partial w}(0,0)=0
$$

and

$$
\frac{\partial^{2} g_{k}}{\partial z \partial w}(0,0) \neq 0 \quad \Longleftrightarrow \quad g_{k}(z, w)=z w \quad \Longleftrightarrow \quad k=1 .
$$

Proceeding in this way, we can show that $\partial \Phi / \partial e_{j}=0$ in $0 \in \ell^{2}$, for all $j \in \mathbb{N}$. Therefore all the derivatives of $\Phi$ vanish at the origin, which means that no regular hypersurface of $\ell^{2}$ can contain a neighborhood of $0 \in X$.

The major advantage of the given definition is that we can recover the local properties of finite-dimensional analytic sets in an infinite dimensional space from the usual local results on analytic sets in a complex manifold. In particular, let $X$ be a finite-dimensional analytic set in $H$, then

1) $X$ admits a local decomposition in finitely many irreducible components;

2) such a decomposition is given by the closure of the connected components of the regular part of $X$;

3 ) for every $x \in X$ there exist a finite-dimensional subspace $L$ of $H$ and an orthogonal projection $\pi: H \rightarrow L$ which realizes a neighborhood of $x \in X$ as a finite covering on $L$;

4) $X$ is locally connected by analytic discs;

5) if $X$ is irreducible, every nonconstant holomorphic function is open;

6) if $X$ is irreducible, the maximum principle holds;

7) if $X$ is compact and $H$ is holomorphically separable, then $X$ is finite.

The behaviour of the analytic sets in a Banach space can vary widely, depending on the properties of the space. We give here some examples.

Example 4.5. Let $c_{0}$ be the vector space of sequences of complex numbers vanishing at infinity, i.e., $\left\{a_{n}\right\} \subset \mathbb{C}$ such that $\lim _{n \rightarrow \infty} a_{n}=0 ; c_{0}$ is a Banach space with the supremum norm. We consider the holomorphic map $f: \mathbb{D} \rightarrow c_{0}$ given by

$$
f(z)=\left\{z^{n}\right\}_{n \in \mathbb{N}}
$$


this $f$ is a regular and injective holomorphic map; its image is contained in the unit ball of $c_{0}$ and if $\left\{z_{k}\right\}$ is a sequence converging to $b \mathbb{D},\left\{f\left(z_{k}\right)\right\}_{k}$ does not converge, therefore $f$ is proper. Thus, $f(\mathbb{D})$ is a complex manifold of dimension 1 in $E$, which is bounded.

Example 4.6. Generalizing the previous example, we consider the Banach space $\ell^{p}$ of $p$-summable sequences of complex numbers, and the holomorphic map $F: \mathbb{D}^{k} \rightarrow$ $\ell^{p}$ given by

$$
F\left(z_{1}, \ldots, z_{k}\right)=\left\{z^{I}\right\}_{I},
$$

where $I$ varies through all the multi-indices of length $k$. We have that

$$
\left|z^{I}\right| \leq\left(\max \left\{\left|z_{1}\right|, \ldots,\left|z_{k}\right|\right\}\right)^{|I|},
$$

and the number of multi-indices $I$ with $|I|=i_{1}+\cdots+i_{k}=m$ is

$$
\left(\begin{array}{c}
m+k-1 \\
k-1
\end{array}\right)
$$

which is less than $(2 m)^{k}$ if $m$ is large enough. Therefore

$$
\sum_{I}\left|z^{I}\right|^{p} \leq \sum_{m}(2 m)^{k}\left(\max \left\{\left|z_{1}\right|, \ldots,\left|z_{k}\right|\right\}\right)^{m}
$$

which converges for $\max \left\{\left|z_{1}\right|, \ldots,\left|z_{k}\right|\right\}<1$.

Again, the map $F$ is regular, injective and proper with unbounded image $F\left(\mathbb{D}^{k}\right)$. We observe that $F\left(\mathbb{D}^{k}\right)$ provides an example of finite dimensional manifold not contained in any finite-dimensional linear subspace.

\subsection{Positive currents}

Definition 4.7. Let $T$ be a metric $(p, p)$-current. We say that $T$ is positive if, given $\pi_{1}, \ldots, \pi_{p} \in \mathcal{O}(X)$,

$$
T\left(f, \pi_{1}, \bar{\pi}_{1}, \ldots, \pi_{p}, \bar{\pi}_{p}\right) \geq 0
$$

for every Lipschitz function $f \geq 0$ on $X$. We say that a current is finitely positive if every finite dimensional projection of it is positive.

Proposition 4.8. Let $\Omega \subset H$ be an open set. $T \in M_{2 p}(\Omega)$ is positive if and only if it is finitely positive.

Proof. Obviously, if $T$ is positive then every complex linear pushforward of $T$ is positive. On the other hand, if $p_{m}: H \rightarrow \mathbb{C}^{m}$ is the projection on the first $m$ coordinates, then $f \circ p_{m} \rightarrow f$ pointwise and $\operatorname{Lip}\left(f \circ p_{m}\right) \leq \operatorname{Lip}(f)$, by Proposition 3.1.

Therefore,

$$
\begin{aligned}
T\left(f, \pi_{1}, \ldots, \bar{\pi}_{p}\right) & =\lim _{m \rightarrow \infty} T\left(f \circ p_{m}, \pi_{1} \circ p_{m}, \ldots, \bar{\pi}_{p} \circ p_{m}\right) \\
& =\lim _{m \rightarrow \infty}\left(p_{m}\right)_{\sharp} T\left(\left.f\right|_{p_{m}(H)},\left.\pi_{1}\right|_{p_{m}(H)}, \ldots,\left.\bar{\pi}_{p}\right|_{p_{m}(H)}\right) \geq 0,
\end{aligned}
$$

which is the thesis. 
On an infinite dimensional complex space, the plurisubharmonic functions are defined by the submean property: let $\Omega \subset H$ be an open set and let $u: \Omega \rightarrow$ $\mathbb{R} \cup\{-\infty\}$ be an upper semicontinuous function (not identically equal to $-\infty$ ); $u$ is plurisubharmonic if

$$
u(a) \leq \int_{0}^{2 \pi} u\left(a+e^{i \theta} b\right) d \theta
$$

for every $a \in \Omega$ and every $b \in H$ such that $a+\lambda b \in \Omega$ for every $\lambda \in \mathbb{C}$ with $|\lambda| \leq 1$. See also [12] for a discussion of the properties of such functions.

Define $d^{c}=i(\partial-\bar{\partial})$.

Proposition 4.9. Let $T \in M_{2 p}(\Omega)$ be a positive closed current with bounded support and $u: \Omega \rightarrow \mathbb{R} \cup\{-\infty\}$ a bounded plurisubharmonic function. Then $d d^{c}(T\llcorner u)$ is a closed, positive metric current with bounded support and the following estimate holds:

$$
M\left(d d^{c}(T\llcorner u)) \leq\|u\|_{\infty} M(T) .\right.
$$

Proof. We note that the result is true for any finite-dimensional projection of $T$. Namely, if $p_{m}$ is as above,

$$
\left(p_{m}\right)_{\sharp} d d^{c}\left(T\llcorner u)=d d^{c}\left(\left(p_{m}\right)_{\sharp} T\left\llcorner\left(u \circ p_{m}\right)\right)=d d^{c}\left(T_{m}\left\llcorner u_{m}\right),\right.\right.\right.
$$

with $T_{m}$ a positive closed current with compact support in $\mathbb{C}^{m}$ and $u_{m}$ a bounded plurisubharmonic function. Then we know that

$$
d d^{c}\left(T_{m}\left\llcorner u_{m}\right)=T_{m}\left\llcorner\left(d d^{c} u_{m}\right)\right.\right.
$$

in the sense of distributions, and for every compact $K$ we have

$$
M_{K}\left(d d^{c}\left(T_{m}\left\llcorner u_{m}\right)\right) \leq C\left\|u_{m}\right\|_{\infty, K} M_{K}\left(T_{m}\right)\right.
$$

As $p_{m}$ is an orthogonal projection, $M_{B}\left(T_{m}\right) \leq M_{B}(T)$, whereas the fact that $T_{m}$ converges weakly to $T$, together with the semicontinuity of the mass, implies that $M_{B}(T) \leq \limsup M_{B}\left(T_{m}\right)$. Therefore, $M_{B}\left(T_{m}\right) \rightarrow M_{B}(T)$ for every bounded set $B$ in $\Omega$.

This means that, for $j$ big enough,

$$
M_{K}\left(d d^{c}\left(T_{m_{j}}\left\llcorner u_{m_{j}}\right)\right) \leq C\|u\|_{\infty, K} M_{K}(T),\right.
$$

so, by Theorem 2.26, we can find a subsequence $w^{*}$-converging to some $S \in$ $M_{2 p-2}(\Omega)$.

Such an $S$ is such that $\left(\pi_{m}\right)_{\sharp} S=T_{m}$ for infinitely many $m$; therefore $S$ coincides, as a metric functional, with $d d^{c}(T\llcorner u)$. Moreover, $S$ is positive and closed, and

$$
M_{K}(S) \leq\|u\|_{\infty, K} M_{K}(T) .
$$

We note also that $\operatorname{supp} S \subseteq \operatorname{supp} T$. 
In view of Proposition 4.9, we will denote by $d d^{c} u \wedge T$ the current $d d^{c}(T\llcorner u)$. Proceeding by induction, we can give a meaning to the writing

$$
d d^{c} u_{1} \wedge \cdots \wedge d d^{c} u_{p} \wedge T
$$

for a current $T$ in the hypotheses of Proposition 4.9. Such a definition allows us to write an analogue of the Monge-Ampère operator in Hilbert spaces.

\subsection{Currents of integration on analytic sets}

Let $V$ be a finite-dimensional analytic set in some open domain $U \subset H$; since, by definition, $V$ is locally contained in some finite-dimensional submanifold, we know that it is locally of finite volume. Therefore, for any $p \in V$ there exists a ball $B$ such that the current $[V]\llcorner B$ of integration on the regular part of $V \cap B$ is a welldefined rectifiable metric current. The following result gives an estimate for the mass of such a current, analogous to Wirtinger formula in the finite dimensional case.

Proposition 4.10. Let $H$ be a Hilbert space, with scalar product $\langle\cdot, \cdot\rangle$, and let $V$ be an analytic set in an open set $U \subset H$, with $\operatorname{dim}_{\mathbb{C}} V_{\mathrm{reg}}=p$. Let $\Omega$ be a ball in $U$ and let $[V]$ be the current of integration associated to $V \cap \Omega$ in $\Omega$. Then

$$
M([V]) \leq \lim _{n \rightarrow \infty} \sum_{1 \leq i_{1}<\cdots<i_{p} \leq n}[V]\left(\frac{i^{p}}{2^{p} p !}, z_{i_{1}}, \bar{z}_{i_{1}}, \ldots, z_{i_{p}}, \bar{z}_{i_{p}}\right)<+\infty,
$$

where $\left\{z_{j}\right\}_{j \in \mathbb{N}}$ are coordinate functions with respect to some orthonormal basis.

Proof. Given an orthonormal basis $\left\{e_{n}\right\}_{n \in \mathbb{N}}$, let $E_{m}=\operatorname{span}\left\{e_{1}, \ldots, e_{m}\right\}$ and $\pi_{m}$ : $H \rightarrow E_{m}$ the orthogonal projection. We denote $[V]_{m}=\left(\pi_{m}\right)_{\sharp}[V]$ and observe that $[V]_{m} \rightarrow[V]$ weakly, so that by the semicontinuity of the mass we get

$$
M([V]) \leq \liminf _{m \rightarrow \infty} M\left([V]_{m}\right),
$$

where the masses are relative to $\Omega$ or to $\Omega_{m}=\Omega \cap E_{m}$ respectively.

On the other hand, the projections $\pi_{m}$ have norm $\left\|\pi_{m}\right\| \leq 1$, so, if $\mu$ is the mass measure of $[V]$, we have

$$
\left|[V]_{m}(f, \eta)\right| \leq \prod \operatorname{Lip}\left(\eta_{j}\right) \int_{U_{m}}|f| d\left(\pi_{m}\right)_{\sharp} \mu .
$$

This implies that the mass measure $\mu_{m}$ of $E_{m}$ is dominated by $\left(\pi_{m}\right)_{\sharp} \mu$, therefore

$$
\mu_{m} \leq\left(\pi_{m}\right)_{\sharp} \mu \underset{m \rightarrow \infty}{\longrightarrow} \mu,
$$

which means that

$$
M([V])=\mu(\Omega) \leq \liminf _{m \rightarrow \infty} \mu_{m}(\Omega) \leq \lim _{m \rightarrow \infty}\left(\pi_{m}\right)_{\sharp} \mu(\Omega)=\mu(\Omega)=M([V]) .
$$


Now, $E_{m}$ with the induced scalar product is the usual complex Hermitian space $\mathbb{C}^{m}$ and the pushforward of an analytic chain is again an analytic chain. Therefore

$$
M\left([V]_{m}\right) \leq[V]_{m}\left(\omega_{m}^{p} / p !\right)=\mathcal{H}^{2 p}\left(V_{m}\right) \leq C_{p} M\left([V]_{m}\right),
$$

where

$$
\omega_{m}=\frac{i}{2} \sum_{j=1}^{m} d z_{j} \wedge d \bar{z}_{j}=\frac{i}{2} \sum_{j=1}^{m} e_{j}^{*} \wedge \bar{e}_{j}^{*}
$$

and $C_{p}$ is a constant depending only on $p$ (see [1], after Remark 8.4). Noticing that $[V]_{m}\left(\omega_{m}^{p} / p !\right)=[V]\left(\omega_{m}^{p} / p !\right)$, we obtain

$$
M([V]) \leq \lim _{n \rightarrow \infty} \sum_{1 \leq i_{1}<\cdots<i_{p} \leq n}[V]\left(\frac{i^{p}}{2^{p} p !}, z_{i_{1}}, \bar{z}_{i_{1}}, \ldots, z_{i_{p}}, \bar{z}_{i_{p}}\right) \leq C_{p} M([V])<+\infty,
$$

which is the thesis.

Remark 4.11. The current $[V]$ is obviously positive, of bidimension $(k, k)$ for some $k$, and its boundary is supported outside $\Omega$.

Given an orthonormal basis $\left\{e_{j}\right\}_{j \in \mathbb{N}}$ and a multiindex $I=\left(i_{1}, \ldots, i_{k}\right)$, let $\pi_{I}$ denote the orthogonal projection from $H$ onto $\operatorname{Span}\left\{e_{i_{1}}, \ldots, e_{i_{k}}\right\}$.

Theorem 4.12. Let $\Omega \subset H$ be a ball, $S$ be an integer rectifiable current in $\Omega$. Suppose that

1) $\operatorname{supp} d S \cap \Omega=\emptyset$;

2) $S$ is a $(k, k)$ positive current.

Then $S$ can be represented as a sum with integer coefficients of integrations on the regular parts of analytic sets.

Remark 4.13. We can not show that $X$ is a finite-dimensional analytic space in the sense specified in the beginning of this section; indeed, in the example discussed before, the map $f(z, w)=\left(z w^{n}\right)_{n}$, gives an analytic space which carries a current of integration which satisfies the hypotheses of the previous theorem but cannot be written as the integration on a finite-dimensional analytic space.

Proof. Since $S$ is a metric current, we can define its pushforward through any Lipschitz map. We note that $\left(\pi_{I}\right)_{\sharp} S=m_{I}\left[V_{I}\right]$ with $V_{I}=\pi_{I}(\Omega)$. By 2$)$, we know that $m_{I} \geq 0$ and, by the fact $S$ is integer rectifiable, $m_{I} \in \mathbb{N}$.

By Theorem 2.15, for almost every $y \in V_{I}$ we can define $\left\langle S, \pi_{I}, y\right\rangle$; moreover, by Theorem 2.13, we can find a countably $\mathcal{H}^{2 k}$-rectifiable subset $B$ of $\operatorname{supp} S$ and an integer multiplicity function $\theta(x)$ such that $S=[B]\llcorner\theta$; then

$$
\left\langle S, \pi_{I}, y\right\rangle=\sum_{x \in \pi_{I}^{-1}(y) \cap B} \theta(x)[x]
$$


and

$$
\sum_{x \in \pi_{I}^{-1}(y) \cap B} \theta(x)=\left\langle S, \pi_{I}, y\right\rangle(1)=m_{I} .
$$

Let us call $G_{I} \subset V_{I}$ the set of $y$ such that the slice exists; then for $j \notin I$ and $z \in G$, we set

$$
P_{j}(z, W)=\prod_{x \in \pi_{I}^{-1}(z) \cap B}\left(W-w_{j}(x)\right)^{\theta(x)},
$$

where $w_{j}(x)$ is the $j$-th coordinate of $x$ in the fixed orthonormal basis.

We note that

$$
\sum_{x \in \pi_{I}^{-1}(z) \cap B} \theta(x) w_{j}(x)^{s}=\left\langle S, \pi_{I}, z\right\rangle\left(w_{j}^{s}\right)
$$

is a holomorphic function of $z$, because $\bar{\partial} S=0$; therefore, by a classical argument, $P_{j}(z, W)$ is a polynomial with coefficients in $\mathcal{O}\left(V_{I}\right)$ for every $j \notin I$.

After removing an $\mathcal{H}^{2 k}$-negligible set from $G_{I}$, we have that $P_{j}\left(z, w_{j}\right)=0$ for every $j \notin I$ and every $x=(z, w) \in \pi_{I}^{-1}\left(G_{I}\right) \cap B$.

Let us define

$$
X_{I}=\left\{P_{j}\left(z, w_{j}\right)=0, j \notin I\right\} \quad \text { and } \quad X=\bigcup_{I} X_{I} .
$$

We can look at $X_{I}$ as the zero locus of the map

$$
P_{I}: H \rightarrow \operatorname{Span}_{\mathbb{C}}\left\{e_{j}: j \notin I\right\}=H_{1}
$$

given by

$$
P_{I}(z, w)=\sum_{j \notin I} e_{j} P_{j}\left(z, w_{j}\right)
$$

In order to show that $P_{I}$ is well defined, we observe that, since $P_{j}(z, W)$ is a polynomial in $W$, for a fixed $z$ we have

$$
\left|P_{j}(z, W)\right| \leq \min \left\{d\left(W, w_{j}(x)\right)^{m_{I}}, x \in \pi_{I}^{-1}(z) \cap B\right\}
$$

with $m \geq 1$, if $W$ is close enough to some $w_{j}(x)$. Therefore, for $p=(z, w)$ in a neighborhood of $B$, we can write

$$
\sum_{j \notin I}\left|P_{j}\left(z, w_{j}\right)\right|^{2} \leq \sum_{j}\left|w_{j}-w_{j}(x)\right|^{2 m_{I}} \leq\|p-x\|^{2 m_{I}}<+\infty,
$$

where $x$ is the nearest point in $B$ to $p=(z, w)$. The map $P_{I}$ is obviously holomorphic, as its entries are polynomials in $w_{j}$ with coefficients holomorphic in the first $k$ coordinates.

This shows that $X_{I}$ is locally given as the zero locus of a holomorphic map between Hilbert space, therefore it is an analytic set. Moreover, let $x \in X_{I}$ be a smooth point and suppose that $\operatorname{dim}_{\mathbb{C}} T_{x} X_{I}>k$. By construction, $\left.\pi_{I}\right|_{T_{x} X_{I}}: T_{x} X_{I}$ $\rightarrow \mathbb{C}^{k}$ has maximum rank, therefore we can find $l \in \mathbb{N}$ such that, setting $J=I \cup\{l\}$, 
the projection $\pi_{J}$ restricted to $T_{x} X_{I}$ is surjective onto $\mathbb{C}^{k+1}$. This means that $P_{m}(z, W) \equiv 0$, but this is impossible. So, $\operatorname{dim}_{\mathbb{C}} T_{x} X_{I}=k$, i.e., the regular part of $X_{I}$ is a smooth $k$-dimensional complex manifold.

Now, let

$$
B_{I}=\left\{x \in B: \mathbf{J}_{2 k} \pi_{I}(x) \neq 0\right\},
$$

i.e., the set of points $x$ of $B$ such that $D \pi_{I}$ has rank $2 k$ on the approximate tangent to $B$ at $x$; define also

$$
C_{I}=(B \cap V) \backslash \pi_{I}^{-1}\left(G_{I}\right)
$$

By Theorem 2.25, we have

$$
\int_{B_{I} \cap C_{I}} \mathbf{J}_{2 k} \pi_{I}(x) d \mathcal{H}^{2 k}(x)=\int_{V_{I} \backslash G_{I}}\left(\int_{\pi_{I}^{-1}(y) \cap B} g d H^{0}\right) d \mathcal{H}^{2 k}(y)=0,
$$

where $g$ is the characteristic function of $B_{I} \cap C_{I}$. Since $J_{2 k} \pi_{I}>0$ on $B_{I} \cap C_{I}$, this means that $H^{2 k}\left(B_{I} \cap C_{I}\right)=0$.

Obviously, $B=\bigcup B_{I}$ and

$$
B \cap\left(\bigcup_{I} \pi_{I}^{-1}\left(G_{I}\right)\right) \subset X
$$

but

$$
B \backslash \bigcup_{I} \pi_{I}^{-1}\left(G_{I}\right)=\bigcap_{I}\left(B \backslash \pi_{I}^{-1}\left(G_{I}\right)\right) \subset \bigcap_{I}\left(\left(B \backslash B_{I}\right) \cup\left(B_{I} \cap C_{I}\right)\right) \subseteq \bigcup_{I}\left(B_{I} \cap C_{I}\right)=D .
$$

Since $\mathcal{H}^{2 k}\left(B_{I} \cap C_{I}\right)=0$, we also have $\mathcal{H}^{2 k}(D)=0$ and $\mathcal{H}^{2 k}(B \backslash X)=0$. Moreover, as $X$ is closed in $\Omega$, supp $S \subset X$; therefore $S=[B \cap X]$.

If we denote by $X_{\text {reg }}$ the union of the regular parts of $X_{I}$, then $S\left\llcorner X_{\text {reg }}\right.$ is a $(k, k)$ current, positive and closed, with support on a $k$-dimensional smooth complex manifold. Therefore, $S\left\llcorner X_{\text {reg }}\right.$ can be written as a series with integer coefficients of the currents of integration on the connected components of $X_{\text {reg. }}$.

There exists $r>0$ such that $\pi_{I}(V)$ contains a ball of radius $r$ for every $I$; therefore, the $\mathcal{H}^{2 k}$-measure of the regular part of $X_{I}$ is uniformly bounded from below independently of $I$. On the other hand $S\left\llcorner X_{\text {reg }}\right.$ is of finite mass; therefore it has to be a finite sum.

Finally, let us consider the rectifiable set $R=B \backslash X_{\text {reg. }}$. If we project it on the first $m$ coordinates, for $m \geq k+1$, we obtain that its image is the singular set of a $k$-dimensional analytic space, therefore $\mathcal{H}^{2 k}$-negligible; again by Theorem 2.25 ,

$$
\int_{R} \mathbf{J}_{2 k}\left(d^{R} \pi_{m x}\right) d \mathcal{H}^{2 k}(x)=\int_{\mathbb{C}^{m}} \sharp\left\{x \in R \cap \pi_{m}^{-1}(y)\right\} d \mathcal{H}^{2 k},
$$

with $\pi_{m}: H \rightarrow \operatorname{Span}\left\{e_{1}, \ldots, e_{m}\right\}$ the orthogonal projection. Let us denote by $\eta(x)$ the approximate tangent to $R$ in $x$; then the $2 k$-Jacobian of $\pi_{m}$ on $\operatorname{Tan}^{(2 k)}(R, x)$ is given by the projection of $\eta(x)$ on $\operatorname{Span}\left\{e_{1}, \ldots, e_{m}\right\}$. 
We define $A_{m}=\left\{x \in R: \mathbf{J}_{2 k}\left(d^{R} \pi_{m x}\right)>0\right\}$ and we note that $A_{k+1} \cup A_{k+2} \cup$ $\cdots=R$, up to $\mathcal{H}^{2 k}$-negligible sets. But

$$
\begin{aligned}
\int_{A_{m}} \mathbf{J}_{2 k}\left(d^{R} \pi_{m x}\right) d \mathcal{H}^{2 k}(x) & =\int_{\mathbb{C}^{m}} \sharp\left\{x \in A_{m} \cap \pi_{m}^{-1}(y)\right\} d \mathcal{H}^{2 k} \\
& =\int_{\pi_{m}\left(A_{m}\right)} \sharp\left\{x \in R \cap \pi_{m}^{-1}(y)\right\} d \mathcal{H}^{2 k}=0
\end{aligned}
$$

because $\pi_{m}\left(A_{m}\right) \subseteq \pi_{m}(R)$, which is $\mathcal{H}^{2 k}$-negligible. Therefore $\mathcal{H}^{2 k}(R)=0$, so $S\left\llcorner X_{\text {reg }}=S\right.$ and this concludes the proof.

\section{Boundaries of holomorphic chains}

In the following definition, we introduce the concept of maximal complexity for a current.

Definition 5.1. Let $S$ be a $(2 p-1)$-current with compact support in a complex manifold $X$; we say that $S$ is maximally complex if $S_{r, s}=0$ for $|r-s|>1$.

As we noted in Section 3, when speaking about the Dolbeault decomposition, the metric functional $S_{r, s}$ always exists, even though it is not always a metric current. However, if it is 0 , then it is obviously a metric current.

The same property for manifolds is stated in terms of their tangent space; namely, a manifold $M$ of real dimension $2 p-1$ is maximally complex if $\operatorname{dim}_{\mathbb{C}} T_{z} M \cap$ $J T_{z} M=p-1$ for every $z \in M$. The first two propositions of this section show that a manifold is maximally complex if and only if the associated current of integration is.

Proposition 5.2. Let $M$ be a compact $\mathcal{C}^{1}$ submanifold of a complex reflexive $B a$ nach space $E$ with complex structure $J$, such that $\operatorname{dim}_{\mathbb{R}} M=2 p-1$ and $\operatorname{dim}_{\mathbb{C}} T_{z} M \cap$ $J T_{z} M=p-1$ for every $z \in M$. Then there exists a complex linear map $F: E \rightarrow \mathbb{C}^{n}$ for some $n>0$ which restricts to an embedding of $M$ into $\mathbb{C}^{n}$.

Proof. Given $z \in M$, let $l_{1, z}, \ldots, l_{p, z}$ linearly independent elements of $E^{*}$ such that

$$
\operatorname{ker} l_{1, z} \cap \cdots \cap \operatorname{ker} l_{p-1, z} \cap T_{z} M \cap J T_{z} M=\{0\}
$$

and

$$
\operatorname{ker} l_{1, z} \cap \cdots \cap \operatorname{ker} l_{p-1, z} \cap \operatorname{ker} \operatorname{Re} l_{p, z} \cap T_{z} M=\{0\} ;
$$

both these conditions are open. By compactness, we can find finitely many $l_{j}$, $j=1, \ldots, N$ such that for every point $z \in M$ there exists indexes $j_{1}<\cdots<j_{p}$ such that

$$
\operatorname{ker} l_{j_{1}} \cap \cdots \cap \operatorname{ker} l_{j_{p-1}} \cap T_{z} M \cap J T_{z} M=\{0\}
$$

and (viewing $E$ as a real vector space)

$$
\operatorname{ker} l_{j_{1}} \cap \cdots \cap \operatorname{ker} l_{j_{p-1}} \cap \operatorname{ker} \operatorname{Re} l_{j_{p}} \cap T_{z} M=\{0\} .
$$


This means that if we define $L: E \rightarrow \mathbb{C}^{N}$ by $L=\left(l_{1}, \ldots, l_{N}\right)$, we have that the differential $d L$ is always of real rank $2 p-1$ on $M$ and it is complex linear on $T_{z} M \cap J T_{z} M$.

Let $U_{1}, \ldots, U_{h}$ be the open sets and $l_{1}, \ldots, l_{p}, l_{p+1}, \ldots, l_{2 p}, \ldots, l_{h p}$ be the maps constructed as above and $\left\{V_{j}\right\}_{j=1}^{K}$ a collection of open sets in $M$ such that for each $V_{j}$ there exists a $U_{\nu(j)}$ such that $V_{j} \Subset U_{\nu(j)}$ and $\bigcup V_{j}=M$.

For a fixed $j$, the set $L^{-1}\left(L\left(V_{j}\right)\right)$ is a union of $\mu_{j}$ connected components which are relatively compact in some open sets $U_{k_{1}}, \ldots, U_{k_{\mu_{j}}}$; therefore, there exist $\mu_{j}$ linear maps $f_{j}^{1}, \ldots, f_{j}^{\mu_{j}}$ such that for each connected component there is one map which separates it from the others, that is, a map which has different values on it and on the union of the others.

Now, consider the map $F=\left(l_{1}, \ldots, l_{h p}, f_{1}^{1}, \ldots, f_{K}^{\mu_{K}}\right)$. By the first part of the construction, $F$ has an injective differential on $M$; by the second part, it is globally injective on $M$. Therefore $F$ is a holomorphic embedding of $M$ into $\mathbb{C}^{n}$, where $n=h p+\mu_{1}+\cdots+\mu_{K}$, realized with a complex linear map.

Let $M$ be a compact $\mathcal{C}^{1}$ submanifold of a reflexive complex Banach space $E$ with complex structure $J$, with $\operatorname{dim}_{\mathbb{R}} M=2 p-1$. $M$ induces a metric current $[M]$ of dimension $2 p-1$.

Proposition 5.3. The following are equivalent:

1) $\operatorname{dim}_{\mathbb{C}}\left(T_{z} M \cap\left(J T_{z} M\right)\right)=p-1$ for all $z \in M$;

2) $[M](\alpha)=0$ for every metric $(r, s)$-form $\alpha$ on $E$ with $r+s=2 p-1$ and $|r-s|>1$;

3) $M$ is locally the graph of a CR-function: for every $z \in M$ there exists a neighbourhood $U$ of $z$ in $E$ such that $M \cap U$ is the graph of a function $f: \widetilde{M} \rightarrow E^{\prime}$, $\widetilde{M}$ a CR-submanifold of $\mathbb{C}^{p}, E^{\prime}$ a closed subspace of $E$ such that $E=E^{\prime} \oplus \mathbb{C}^{p}$ and $f$ a $C R$-function on $\widetilde{M}$.

Proof. 1) $\Longrightarrow 2)$ Let $\alpha=\left(f, g_{1}, \ldots, g_{r}, h_{1}, \ldots, h_{s}\right)$ and let $i: M \rightarrow E$ be an embedding whose differential is complex linear when restricted to (the preimage of) $T_{z} M \cap J T_{z} M$; then $[M]=i_{\sharp} T$, with $T \in M_{2 p-1}(M)$. By the comparison theorem for manifolds, $T$ is induced by a classical current $T^{\prime}$ on $M$; but then,

$$
[M](\alpha)=T\left(f \circ i, g_{1} \circ i, \ldots, h_{s} \circ i\right)=T^{\prime}\left(f \circ i d\left(g_{1} \circ i\right) \wedge \cdots \wedge d\left(h_{s} \circ i\right)\right)
$$

The functions $g_{j} \circ i$ have complex linear differentials on $i_{*}^{-1}(T M \cap J T M)$; therefore, if there are more than $p$ of them, their wedge product will vanish. The same holds for the differentials of the functions $\bar{h}_{j} \circ i$. So $[M](\alpha)=0$ if $|r-s|>1$.

$2) \Longrightarrow 1$ ) Let $\rho: E \rightarrow \mathbb{C}^{N}$ be a finite-dimensional embedding for $M$, which is holomorphic on $E$. If

$$
\operatorname{dim}_{\mathbb{C}} T_{\rho(z)} \rho(M) \cap J_{\mathbb{C}^{N}} T_{\rho(z)} \rho(M)=\operatorname{dim}_{\mathbb{C}} T_{z} M \cap J T_{z} M<p-1,
$$


then there exists a metric $(r, s)$-form $\beta$ on $\mathbb{C}^{N}$ with $r+s=2 p-1$ and $|r-s|>1$ such that

$$
\int_{\rho(M)} \beta \neq 0,
$$

SO

$$
\int_{M} \rho^{*} \beta \neq 0
$$

and $\rho^{*} \beta$ is a $(r, s)$-form on $E$ with $|r-s|>1$.

$3) \Longrightarrow 1$ ) Let $f: \widetilde{M} \rightarrow E^{\prime}$ be the given CR-function; we define $G: \widetilde{M} \rightarrow \widetilde{M} \times E^{\prime}$ by $G(p)=(p, f(p))$.

Let $F: \mathbb{C}^{p} \oplus E^{\prime} \rightarrow E$ be the given isomorphism; then $(F \circ G)_{*} T_{p} \widetilde{M}=T_{F(p, f(p))} M$ and, since $T_{p} \widetilde{M}$ contains a complex subspace of complex dimension $p-1$, so does the tangent space of $M$.

1) $\Longrightarrow 3$ ) Let us fix $z \in M$ and let $H_{z}$ be the complex subspace of $T_{z} E$ of (complex) dimension $p$ containing $T_{z} M$. By reflexivity, $E=E^{*}$, so we have a splitting of $E=H_{z} \oplus E^{\prime}$ for some closed subspace $E^{\prime}$. By construction, $\pi: E \rightarrow H_{z}$ is a local embedding when restricted to a neighbourhood $U$ of $z$ in $M$, because it has a maximum rank differential at $z$.

Let $\widetilde{M}$ be the image of $U$ trough $\pi$; we have the function $f: \widetilde{M} \rightarrow E^{\prime}$ defined by $(p, f(p)) \in U \cap \pi^{-1}(p)$. By construction, $\left.f_{*}\right|_{T_{p} \widetilde{M} \cap J T_{p} \widetilde{M}}$ is $\mathbb{C}$-linear, so $f$ is a CR-function and $U$ is its graph.

Proposition 5.4. Let $M$ be a $(2 p-1)$-current with compact support in $X$, and let $F: X \rightarrow Y$ be a Lipschitz holomorphic map. Suppose that $M$ is maximally complex. Then the same is true for $F_{\sharp} M$ and, if $p>\operatorname{dim}_{\mathbb{C}} Y$, for $\langle M, F, \zeta\rangle$, given that $M$ is flat and slices exist.

Proof. Obviously, we have $\left(F_{\sharp} M\right)_{r, s}=F_{\sharp}\left(M_{r, s}\right)$ (this is an equality between metric functionals only, not metric currents).

Moreover, if $\operatorname{dim}_{\mathbb{C}} Y<p$ and if $\langle M, F, \zeta\rangle$ exists for some $\zeta \in Y$, let $\left\{\rho_{\epsilon, \zeta}\right\}$ be a family of smooth approximations of $\delta_{\zeta}$. Then locally (with supp $f$ contained in a manifold chart for $Y$ ),

$$
\langle M, F, \zeta\rangle(f, \eta)=\lim _{\epsilon \rightarrow 0} M\left(f \rho_{\epsilon, \zeta}, F, \bar{F}, \eta\right) .
$$

So, if $M_{r, s}=0$ for $|r-s|>1$ then also $\langle M, F, \zeta\rangle_{r-m, s-m}=0$ for $1<|r-s|=$ $|(r-m)-(s-m)|$, with $m=\operatorname{dim}_{\mathbb{C}} Y$.

Definition 5.5. A $M C$-cycle in a complex Banach space $E$ is a maximally complex $(2 p-1)$-dimensional closed metric current, with compact support.

Remark 5.6. The definition is meaningless for $p=1$; the notion of moment condition which substitutes the maximal complexity for 1-dimensional currents cannot be given that easily in a Banach space and it turns out to be not automatically satisfied by a maximally complex current of higher dimension. The philosophical reason is the greater distance, in Banach spaces, between local and global aspects. 
The following theorem follows easily from Proposition 5.4 and from the slicing properties of rectifiable currents.

Theorem 5.7. Let $M$ be a rectifiable $M C$-cycle of dimension $(2 p-1)$ in a Banach space $E$ and consider a Lipschitz holomorphic map $F: E \rightarrow \mathbb{C}^{m}$. Then

1) $F_{\sharp} M$ is a rectifiable $M C$-cycle of dimension $(2 p-1)$ in $\mathbb{C}^{m}$;

$2)$ if $m<p-1,\langle M, F, \zeta\rangle$ is a rectifiable $M C$-cycle of dimension $2(p-m)-1$ in $E$.

Remark 5.8. By Theorem 2.15, the slice $\langle M, F, \zeta\rangle$ exists and is rectifiable for almost every $\zeta \in \mathbb{C}^{m}$.

Theorem 5.9. Let $M$ be a MC-cycle of dimension $(2 p-1)$ in $E$. Then, for every linear projection $\pi: E \rightarrow \mathbb{C}^{p}$ and every $\phi \in \mathcal{O}(\overline{\operatorname{supp} M})$, we have

$$
\bar{\partial}\left[\pi_{\sharp}(\phi M)\right]^{0,1}=0 .
$$

Moreover, there is a unique integrable compactly supported function $c_{\phi}$ in $\mathbb{C}^{p}$ such that

$$
\bar{\partial} c_{\phi}=\left[\pi_{\sharp}(\phi M)\right]^{0,1},
$$

and such a function can be obtained by convolution with the Cauchy kernel or the Bochner-Martinelli kernel.

Proof. We know that $d M=0$; since $M$ is maximally complex, we have

$$
M=M_{p, p-1}+M_{p-1, p}
$$

so

$$
0=d M=(d M)_{p-2, p}+(d M)_{p-1, p-1}+(d M)_{p, p-2} .
$$

In particular, this means that $(d M)_{p, p-2}=0$. Therefore,

$$
\begin{aligned}
\bar{\partial}\left[\pi_{\sharp}(\phi M)\right]^{0,1} & =\bar{\partial}\left[\pi_{\sharp}(\phi M)\right]_{p, p-1}=\left[d \pi_{\sharp}(\phi M)\right]_{p, p-2}=\left[\pi_{\sharp}(d \phi M)\right]_{p, p-2} \\
& =\left[\pi_{\sharp}(\phi d M)\right]_{p, p-2}+\left[\pi_{\sharp}(M\llcorner(1, \phi))]_{p, p-2},\right.
\end{aligned}
$$

but $M\llcorner(1, \phi)$ has non-vanishing $(r, s)$-components only for $(r, s)=(p-1, p-1)$ or $(r, s)=(p-2, p-1)$, so $\left[\pi_{\sharp}(M\llcorner(1, \phi))]_{p, p-2}=0\right.$. Then,

$$
\bar{\partial}\left[\pi_{\sharp}(\phi M)\right]^{0,1}=\left[\pi_{\sharp}(\phi d M)\right]_{p, p-2}=\pi_{\sharp}\left(\phi(d M)_{p, p-2}\right)=0 .
$$

We note that $\pi_{\sharp}(\phi M)$ is a metric current in $\mathbb{C}^{p}$, therefore it is also a classical one, consequently its component of bidegree $(0,1)$ is a classical current as well and by the previous computation is $\bar{\partial}$-closed. By a standard convolution-contraction with either the Cauchy kernel or the Bochner-Martinelli kernel, we can find a compactly supported integrable function $c_{\phi}$ as requested. 
Theorem 5.10. Let $M$ be a compact, oriented $(2 p-1)$-manifold (without boundary) of class $\mathcal{C}^{2}$ embedded in $H$, and suppose that there exists an orthogonal decomposition $H=\mathbb{C}^{p} \oplus H^{\prime}$ such that the projection $\pi: H \rightarrow \mathbb{C}^{p}$, when restricted to $M$, is an immersion with transverse self-intersections. Then, if $M$ is maximally complex (i.e., $[M]$ is an MC-cycle), there exists a unique holomorphic p-chain $T$ in $H \backslash M$ with $\operatorname{supp} T \Subset H$ and finite mass, such that $d T=[M]$ in $H$.

Proof. Let $\mathfrak{m}=\pi(M) \subset \mathbb{C}^{p}$; for every $\lambda \in H^{\prime} \backslash\{0\}$, we define $\pi^{\lambda}(z)=(\pi(z),\langle z, \lambda\rangle)$ $\in \mathbb{C}^{p+1}$.

By the previous results, $M^{\lambda}$ satisfies the same hypotheses in $\mathbb{C}^{p+1}$. Therefore, by Theorem 6.1 in [6], we can solve the problem for $M^{\lambda}=\pi^{\lambda}(M)$, finding a holomorphic $p$-chain $T^{\lambda}$ in $\mathbb{C}^{p+1} \backslash M$ with the required properties. Following the proof of Theorem 6.1 in [6], we write

$$
\mathbb{C}^{p} \backslash \mathfrak{m}=U_{0} \cup U_{1} \cup \cdots \cup U_{k},
$$

where the $U_{j}$ are connected components and $U_{0}$ is unbounded; $T^{\lambda}$ is locally on each $U_{j}$ union of graphs of holomorphic functions

$$
F_{j}^{\lambda, h}: U_{j} \rightarrow \mathbb{C}, \quad h=1, \ldots, n_{\lambda, j} .
$$

Given another $\lambda^{\prime} \in H^{\prime} \backslash\{0\}$, we can consider the $p$-chain $T^{\lambda^{\prime}}$, which will be given by holomorphic functions

$$
F_{j}^{\lambda^{\prime}, h}: U_{j} \rightarrow \mathbb{C}, \quad h=1, \ldots, n_{\lambda^{\prime}, j} .
$$

However, we can also consider, in $\mathbb{C}^{p+2}$, the manifold $M^{\lambda, \lambda^{\prime}}$ and the associated solution $T^{\lambda, \lambda^{\prime}}$; denoting by $p$ and $p^{\prime}$ the restrictions of $\pi^{\lambda}$ and $\pi^{\lambda^{\prime}}$ to $\mathbb{C}^{p+2}$, we have

$$
p_{*} T^{\lambda, \lambda^{\prime}}=T^{\lambda} \quad \text { and } \quad p_{*}^{\prime} T^{\lambda, \lambda^{\prime}}=T^{\lambda^{\prime}} .
$$

Since the differentials of $p$ and $p^{\prime}$ are of rank $2 p-1$ on $M^{\lambda, \lambda^{\prime}}$, and because $p$ and $p^{\prime}$ are holomorphic, their differentials are at least of rank $2 p$ on $M^{\lambda, \lambda^{\prime}}$; this means that they are of rank $2 p$ in a neighborhood of $M^{\lambda, \lambda^{\prime}}$ in $M^{\lambda, \lambda^{\prime}} \cup \operatorname{supp} T^{\lambda, \lambda^{\prime}}$ (which is locally a $\mathcal{C}^{2}$ manifold with boundary by Lemma 6.8 in [6]). Therefore, $n_{\lambda, j}=n_{\lambda, \lambda^{\prime}, j}=n_{\lambda^{\prime}, j}$ for every $j$ and every $\lambda, \lambda^{\prime} \in H^{\prime} \backslash\{0\}$.

Let $\left\{\lambda_{i}\right\}_{i \in I}$ be an orthonormal basis for $H^{\prime}$ and consider the holomorphic functions

and define

$$
F_{j}^{\lambda_{i}, h}: U_{j} \rightarrow \mathbb{C}, \quad j=1, \ldots, k, \quad h=1, \ldots, n_{j}, \quad i \in I,
$$

$$
F_{j}^{h}=\sum_{i \in I} \lambda_{i} F_{j}^{\lambda_{i}, h}
$$

The function $F_{j}^{h}$ is well defined. For any finite subset of indices $J \subset I$, we can consider the projection

$$
p_{J}: H \rightarrow \mathbb{C}^{p} \oplus \operatorname{Span}\left\{\lambda_{i}\right\}_{i \in J}
$$

and the pushforward $[M]_{J}=\left(p_{J}\right)_{\sharp}[M]$. 
The functions $\left\{F_{j}^{\lambda_{i}, h}\right\}_{i \in J}$ give a solution for the finite-dimensional problem with datum $[M]_{J}$, therefore $S_{J, j, h}=\sum_{i \in J} F_{j}^{\lambda_{i}, h} \lambda_{i}$ is a holomorphic function, with values in a finite-dimensional vector space, such that

$$
\left|S_{J, j, h}(z)\right| \leq R,
$$

where $R$ is such that $\operatorname{supp}[M]_{J} \subset \mathbb{C}^{p} \times B(0, R), B(z, r)$ being the ball with center $z$ and radius $r$ in $\operatorname{Span}\left\{\lambda_{i}\right\}_{i \in J}$.

Now, let us take $I=\mathbb{N}$ and fix $\epsilon>0$. By compactness, we can find $I^{\prime} \subset I$ finite and set

$$
V_{\epsilon}=\mathbb{C}^{p} \oplus \operatorname{Span}\left\{\lambda_{i}\right\}_{i \in I^{\prime}}
$$

so that $d\left(M, V_{\epsilon}\right)<\epsilon$; let $H_{\epsilon}^{\prime}$ be the topological complement of $V_{\epsilon}$ in $H$, then the projection of $M$ on $H_{\epsilon}^{\prime}$ lies in a ball of radius $\epsilon$ around 0 . Now, for any finite subset $J \subset I$ such that $\min J>\max I^{\prime}$, we have that

$$
\left|S_{J, j, h}(z)\right| \leq \epsilon,
$$

showing that the sequence of maps from $U_{j}$ to $H^{\prime}$

$$
\left\{\sum_{i=0}^{m} F_{j}^{\lambda_{i}, h}(z) \lambda_{i}\right\}_{m \in I}
$$

is a Cauchy sequence with respect to the supremum norm on $U_{j}$. Therefore the limit $F_{j}^{h}(z)$ is well defined and continuous on the closure of $U_{j}$, because every element of the sequence is.

The function $F_{j}^{h}$ is holomorphic. Indeed, for any $\lambda \in H^{\prime}$, we write

$$
\lambda=\sum_{i \in I} \alpha_{i} \lambda_{i} \quad \text { and } \quad\left\langle F_{j}^{h}(z), \lambda\right\rangle=\sum_{i \in I} \alpha_{i} F_{j}^{\lambda_{i}, h}(z) .
$$

We now observe that

$$
\begin{gathered}
\left|\sum_{i \in I} \alpha_{i} F_{j}^{\lambda_{i}, h}(z)\right| \leq \sqrt{\sum_{i \in I}\left|F_{j}^{\lambda_{i}, h}\right|^{2}} \sqrt{\sum_{i \in I}\left|\alpha_{i}\right|^{2}} \leq\|\lambda\|_{H^{\prime}} \sqrt{\sum_{i \in I}\left|F_{j}^{\lambda_{i}, h}(z)\right|_{\infty, U_{j}}^{2}} \\
<\|\lambda\|_{H^{\prime}}\left\|\left|F_{h}^{j}\right|\right\|_{\infty, U_{j}},
\end{gathered}
$$

which is finite, and this implies that the sequence of holomorphic functions

$$
\left\{\sum_{i=0}^{m} \alpha_{i} F_{j}^{\lambda_{i}, h}\right\}_{m \in I}
$$

converges uniformly on $U_{j}$. The limit is then holomorphic, so $F_{j}^{h}$ is holomorphic.

The function $F_{j}^{h}$ extends $\mathcal{C}^{1}$ to the boundary. By [6], there exist sets $A \subset \mathfrak{m}$ and $A_{i} \subset M^{\lambda_{i}}$ with $\pi\left(A_{i}\right)=A$, which are $\mathcal{H}^{2 p-1}$-negligible and such that outside them we have $\mathcal{C}^{1}$ regularity for $\operatorname{supp} T^{\lambda_{i}} \cup M^{\lambda_{i}}$ and for the functions $F_{j}^{\lambda_{i}, h}$. Let us consider $p \in \mathfrak{m} \cap \overline{U_{j}} \backslash A$; for each $i \in I$, one of the following two cases can occur: 
1) $F_{j}^{\lambda_{i}, h}(p) \notin M^{\lambda_{i}}$

2) $F_{j}^{\lambda_{i}, h}(p) \in M^{\lambda_{i}}$.

In the former, $F_{j}^{\lambda_{i}, h}$ extends holomorphically through $p$, whereas in the latter we can find a relatively compact neighborhood $V$ of $p$ in $\mathfrak{m}$ such that $F_{j}^{\lambda_{i}, h}$ coincides on $V$ with some $C R$ function $f: V \rightarrow M^{\lambda_{i}}$. In both cases, $F_{j}^{\lambda_{i}, h}$ is of class $\mathcal{C}^{1}$ near $p$. Let $U$ be an open set with $\mathcal{C}^{1}$ boundary in $U_{j}$ such that $b U_{j} \cap b U=V$.

The restrictions of the derivatives of $F_{j}^{h}$ to $b U_{j}$ are continuous, when we derive in a direction tangent to $T b U$; however, by the Cauchy-Riemann equations, we can control the normal derivative with the tangential ones, therefore also the normal derivative of $F_{j}^{h}$ is a continuous function when restricted to $b U$.

We note that from this follows that the image of $b U$ through one of these maps is a compact set in $H^{\prime}$ and we can replicate the previous argument, obtaining that the sequence

$$
\left\{\sum_{i=0}^{m} \frac{\partial}{\partial z_{s}} F_{j}^{\lambda_{i}, h}(z) \lambda_{i}\right\}_{m \in I}
$$

is a Cauchy sequence with respect to the supremum norm on $U$.

Therefore, the limit is continous on the closure of $U$, thus implying that

$$
\left\|\left|\frac{\partial}{\partial z_{s}} F_{j}^{h}\right|\right\|_{\infty, U}<+\infty .
$$

Moreover, on $b U \cap b U_{j}=V, F_{j}^{h}$ coincides with $f$ and we can cover $\mathcal{H}^{2 p-1}$-almost all of $b U_{j}$ with open sets where $F_{j}^{h}$ coincides with some CR-functions realizing $M$ as a graph. Therefore, as $M$ is a compact $\mathcal{C}^{1}$ manifold,

$$
\left\|\left|\frac{\partial}{\partial z_{s}} F_{j}^{h}\right|\right\|_{\infty, b U_{j}}<+\infty
$$

and hence

$$
\left\|\left|\frac{\partial}{\partial z_{s}} F_{j}^{h}\right|\right\|_{\infty, U_{j}}<+\infty .
$$

The current of integration on the graph of $F_{j}^{h}$ has finite mass. By the previous paragraph, there exists a constant $C_{h, j}$ such that

$$
\left|\nabla F_{j}^{h}(z)\right|^{2}=\sum_{i \in I}\left|\nabla F_{j}^{\lambda_{i}, h}(z)\right|^{2} \leq C_{h, j} \quad \text { for every } z \in U_{j} .
$$

It is easy to show that there exists a polinomial $g_{p}(X)$ such that

$$
\sum_{i}\left|a_{i}\right| \leq S<+\infty \Longrightarrow \sum_{|J|=p} \prod_{i \in J}\left|a_{i}\right| \leq g_{p}(S)<+\infty .
$$

Therefore,

$$
\sum_{|J|=p} \prod_{i \in J}\left|\nabla F_{j}^{\lambda_{i}, h}(z)\right| \leq g_{p}\left(C_{h, j}\right)<+\infty \quad \text { for every } z \in U_{j} .
$$


We consider the $(p, p)$-form

$$
\eta_{j}^{h}(z)=\sum_{|J|=p} \bigwedge_{i \in J} d F_{j}^{\lambda_{i}, h}(z) \wedge d \bar{F}_{j}^{\lambda_{i}, h}(z),
$$

which is well-defined by the previous estimates, and note that

$$
\left\|\eta_{j}^{h}\right\|_{\infty, U_{j}} \leq g_{p}\left(C_{h, j}\right)
$$

Let $\left\{w_{i}\right\}_{i \in I}$ be coordinates for the basis $\left\{\lambda_{i}\right\}_{i \in I}$, i.e., $w_{i}(v)=\left\langle v, \lambda_{i}\right\rangle$ for $v \in H^{\prime}$, and denote by $T_{h, j}$ the (alleged) current of integration on the graph of $F_{j}^{h}$. Then

$$
\begin{aligned}
& T_{h, j}\left(1, w_{i_{1}}, \bar{w}_{i_{1}}, \ldots, w_{i_{p}}, \bar{w}_{i, p}\right) \\
& \quad=\int_{U_{j}} d F_{j}^{\lambda_{i_{1}}, h}(z) \wedge d \bar{F}_{j}^{\lambda_{i_{1}}, h}(z) \wedge \cdots \wedge d F_{j}^{\lambda_{i_{p}}, h}(z) \wedge d \bar{F}_{j}^{\lambda_{i_{p}}, h}(z) .
\end{aligned}
$$

Therefore, by Proposition 4.10, we have

$$
M\left(T_{h, j}\right) \leq \mathcal{L}^{2 p}\left(U_{j}\right) \sum_{p^{\prime}=0}^{p} g_{p^{\prime}}\left(C_{h, j}\right)<+\infty .
$$

We have to sum all the values from 0 to $p^{\prime}$ because we apply the formula of Proposition 4.10 in $H$ and not in $H^{\prime}$, so we have to consider also the $p$-tuples of coordinates coming in part from $\mathbb{C}^{p}$ and in part from $H^{\prime}$.

As the $F_{j}^{h}$ are a finite number of functions, we can consider the metric functional of integration on their graphs and denote it by $T$. $T$ is a holomorphic $p$-chain in $H \backslash M$, it has finite mass and its support is contained in a product of discs, therefore it is relatively compact in $H$. Moreover, for $\mathcal{H}^{2 p-1}$-almost every point in $M$ there is a neighborhood where $\operatorname{supp} T \cup M$ is a $\mathcal{C}^{1}$ manifold.

This implies that $T$ is a metric rectifiable $(p, p)$-current in $H$. We note that for any finite-dimensional projection $p: H \rightarrow \mathbb{C}^{m}$, we have that $d\left(p_{\sharp} T\right)=p_{\sharp}[M]$; it is an easy application of Theorem 3.3 to show that this implies $d T=[M]$. Finally, it is not difficult to see that the map $x \mapsto\left(x, F_{j}^{h}(x)\right)$ is proper into $H \backslash M$, which is an hilbertian manifold, hence by Theorem 4.3 its image is a finite dimensional complex space in $H \backslash M$.

Remark 5.11. Suppose we are given a family $M_{s}$ of $M C$-cycles each satisfying the hypothesis of Theorem 5.10, depending on some parameter $s \in U \subset \mathbb{C}$ in a $\mathcal{C}^{1}$ way. Locally in $s$, we can assume that the various manifolds $M_{s}$ project, through the maps $\pi_{s}$ given by hypothesis, to the same immersed manifold $\mathfrak{m} \subset \mathbb{C}^{p}$.

Therefore, the functions $F_{j, s}^{\lambda, h}$ constructed during the proof of Theorem 5.10 depend on $s$ in a $\mathcal{C}^{1}$ way. This implies that $F_{j, s}^{h}$ vary Lipschitz-continuously in $s$.

Hence, the map associating to $s$ the solution to $d T_{s}=\left[M_{s}\right]$ is Lipschitzcontinuous in $s$.

The compactness of $M$ is needed only to ensure that there is a finite number of connected components in $\mathbb{C}^{p} \backslash \mathfrak{m}$. Therefore, we also have the following result. 
Theorem 5.12. Let $M$ be a bounded, oriented ( $2 p-1)$-manifold (without boundary) of class $\mathcal{C}^{2}$ embedded in $H$, with finite volume. Assume that there exists an orthogonal decomposition $H=\mathbb{C}^{p} \oplus H^{\prime}$ such that the projection $\pi: H \rightarrow \mathbb{C}^{p}$, when restricted to $M$, is a closed immersion with transverse self-intersections. Then, if $[M]$ is an $M C$-cycle, there exists a unique holomorphic p-chain $T$ in $H \backslash M$ with $\operatorname{supp} T \Subset H$ and finite mass, such that $d T=[M]$ in $H$.

Proof. As $\pi$ is supposed to be closed, $\pi(M)$ is a closed and bounded subset of $\mathbb{C}^{p}$, therefore compact. By the finiteness of volume, we know that $[M]$ is a well-defined metric current and we can proceed with the same proof as before.

Remark 5.13. By Definition 4.1, there exists a finite dimensional complex submanifold $V$ of $H \backslash M$ containing the support of the holomorphic chain $T$. Therefore, we recover all the results that are true in finite dimension, e.g. if $M$ is contained in a strictly pseudoconvex hypersurface, we know that $T$ will have only finitely many singular points.

\section{References}

[1] Ambrosio, L. And Kirchheim, B.: Currents in metric spaces. Acta Math. 185 (2000), no. 1, 1-80.

[2] Ambrosio, L. And Kirchieim, B.: Rectifiable sets in metric and Banach spaces. Math. Ann. 318 (2000), no. 3, 527-555.

[3] Ambrosio, L. And Schmidt, T.: Compactness results for normal currents and the Plateau problem in dual Banach spaces. Proc. Lond. Math. Soc. (3) 106 (2013), no. $5,1121-1142$.

[4] Aurich, V.: Local analytic geometry in Banach spaces. In Complex analysis, functional analysis and approximation theory (Campinas, 1984), 1-23. North-Holland Math. Stud. 125, North-Holland, Amsterdam, 1986.

[5] DouAdy, A.: Le problème des modules pour les sous-espaces analytiques compacts d'un espace analytique donné. Ann. Inst. Fourier (Grenoble) 16 (1966), fasc. 1, 1-95.

[6] Harvey, F. R. and Lawson, JR., H. B.: On boundaries of complex analytic varieties. I. Ann. of Math. (2) 102 (1975), no. 2, pp. 223-290.

[7] Harvey, F. R. and Lawson, JR., H. B.: On boundaries of complex analytic varieties. II. Ann. of Math. (2) 106 (1977), no. 2, pp. 213-238.

[8] Harvey, R. and Shiffman, B.: A characterization of holomorphic chains. Ann. of Math. (2) 99 (1974), 553-587.

[9] King, J. R.: The currents defined by analytic varieties. Acta Math. 127 (1971), no. 3-4, 185-220.

[10] Mongodi, S.: Some applications of metric currents to complex analysis. Manuscripta Math. 141 (2013), no. 3-4, 363-390.

[11] Mongodi, S. And Saracco, A.: Non compact boundaries of complex analytic varieties in Hilbert spaces. Complex Manifolds 1 (2014), no. 1, 34-44.

[12] MujicA, J.: Complex analysis in Banach spaces. In Holomorphic functions and domains of holomorphy in finite and infinite dimensions. North-Holland Mathematics Studies 120, North-Holland, Amsterdam, 1986. 
[13] Noverraz, P.: Pseudo-convexité, convexité polynomiale et domaines d'holomorphie en dimension infinie. North-Holland Mathematics Studies 3, North-Holland, Amsterdam, 1973.

[14] Ruget, G.: À propos des cycles analytiques de dimension infinie. Invent. Math. 8 (1969), 267-312.

[15] Shiffman, B.: On the removal of singularities of analytic sets. Michigan Math. J. 15 (1968), 111-120.

Received October 31, 2013; revised March 13, 2014.

Samuele Mongodi: Dipartimento di Matematica, Università di Roma "Tor Vergata", Via della Ricerca Scientifica, I-00133 Roma, Italy.

E-mail: mongodi@mat.uniroma2.it, samuele.mongodi@gmail.com

The author acknowledges the support of the Italian project FIRB-IDEAS "Analysis and Beyond". 